\title{
Hörimplantate im Zeitalter der Digitalisierung
}

\section{Hearing Implants in the Era of Digitization}

\section{(ㄷ) (i) (우)}

Autoren

Heidi Olze, Florian Cornelius Uecker, Sophia Marie Häußler, Steffen Knopke, Agnieszka J. Szczepek, Stefan Gräbel

Institut

Klinik für Hals-, Nasen- und Ohrenheilkunde CVK, Klinik und Poliklinik für Hals-, Nasen-, Ohrenheilkunde CCM, Charité Universitätsmedizin Berlin

\section{Schlüsselwörter:}

Cochlea Implantat, Remote Care, Telemedizin, App, BigData, Artificial Intelligence

\section{Bibliografie}

DOI https://doi.org/10.1055/a-0755-2811

Laryngo-Rhino-Otol 2019; 98: S82-S105

(c) Georg Thieme Verlag KG Stuttgart · New York

ISSN 0935-8943

Korrespondenzadresse

Prof. Dr. med. Heidi Olze

Charité-Universitätsmedizin-Berlin

CVK: Augustenburger Platz 1, D-13353 Berlin

CCM: Charitéplatz 1, D-10117 Berlin

Heidi.Olze@charite.de

\section{ZUSAMMENFASSUNG}

Die Entwicklungen der letzten Jahre haben gezeigt, dass die Rehabilitation mit Hörimplantaten in der HNO-Heilkunde ein Bereich mit höchstem Entwicklungs- und Innovationspotenzial ist. Neue, bzw. erweiterte Indikationen gingen dabei einher mit Entwicklungen im Bereich der Implantate, neuen OP-Techniken und entsprechend angepassten Rehabilitationsstrategien.

Die wachsende Anzahl von Menschen mit Hörstörungen, die erweiterten Indikationen und die daraus resultierende steigende Anzahl Cl-Versorgter, sowie die Notwendigkeit der lebenslangen CI Nachsorge sind vor dem Hintergrund begrenzter Ressourcen eine große Herausforderung. Um diese zu bewältigen und zu gestalten, bedarf es neben einer engen interdisziplinären Zusammenarbeit und kontinuierlichen Weiterentwicklung der Therapie auch ganz neuer Strategien. Hier kommt der Digitalisierung all dieser Prozesse eine Schlüsselrolle zu.

In diesem Referat werden die aktuellen Entwicklungen aus dem Blickwinkel einer Cochlea-Implantat $(\mathrm{Cl})$ versorgenden Klinik dargestellt und besprochen. Das Referat illustriert vielfältige digitale Anwendungen, die in allen Phasen der CI Versorgung zum Einsatz kommen können, angefangen von der Patienteninformation über Möglichkeiten des Hör-Screenings und die präoperative Evaluation bis zur lebenslangen Nachsorge und der klinischen Forschung.

Hierbei liegt der Fokus auf speziellen Applikationen, die in der Entwicklung des digitalen Fortschritts und digitaler Strukturen im Rahmen der CI Versorgung eine besondere Rolle spielen und für das Verständnis der weiteren Entwicklung wichtig sind.

Möglichkeiten einer vereinfachten Anpassung ergeben sich z. B. durch eine automatische MAP-Erstellung (Artificial Intelligence Anwendungen),
Remote Care Netzwerke (Telemedizin, Apps) fördern die aktive Selbstbeteiligung der Patienten und erlauben völlig neue Formen einer ortsungebundenen Patientenversorgung (automatisierte technische Implantatkontrolle, Selbstprogrammierung, Upgrades). Zentrale Datenbanken können die aktuelle MAP z. B. im Reparaturfall speichern, technische Daten und die Hörleistung dokumentieren. Einige der skizzierten Anwendungen sind heute schon Realität, andere erst in der Entwicklung. Das Verständnis über die Möglichkeiten der Digitalisierung und deren Einsatz im Bereich der Hörrehabilitation mit Hörimplantaten und die Erkenntnis über das enorme Potenzial für effektive, zeiteffiziente Strukturen sind unabdingbar, um dieses Potenzial zu nutzen. Für uns HNO-Ärzte als wichtige Akteure im Gesundheitssystem bedeutet das, dass wir neben einer hohen fachlichen Expertise auch hohen Anforderungen an unsere Qualifikation in Bezug auf die digitalen Anwendungen gerecht werden müssen, um diesen Prozess aktiv mitzugestalten.

\section{ABSTRACT}

The last years' developments could show that the rehabilitation with hearing implants is a field with the highest potential for development and innovation in otorhinolaryngology. New or extended indications were seen with developments of implants, new surgical techniques, and respective rehabilitation strategies.

With the background of limited resources, the increasing number of subjects suffering from hearing disorders, the extended indications and thus the increasing number of $\mathrm{Cl}$ carriers as well as the need of life-long Cl follow-up are one of the major challenges of the future. In order to cope with this situation, completely new strategies are required beside a close interdisciplinary cooperation and continuous development of the therapy. In this context, digitization of all these processes plays a key role. This manuscript will describe and discuss the current developments from the perspective of a cochlea implant $(\mathrm{Cl})$ providing hospital. The contribution will elucidate manifold digital applications that may be implemented in all phases of $\mathrm{Cl}$ provision, starting with patient information about the possibilities of hearing screening and preoperative evaluation up to life-long follow-up and clinical research.

The focus is mainly placed on specific applications that play a particular role in the development of digital progresses and structures in the context of cochlea implantation and that are crucial for understanding the further development.

The options of simplified fitting result for example from automated MAP creation (artificial intelligence); remote care networks (telemedicine, apps) foster the active contribution of the patients themselves and allow completely new types of location-independent healthcare (automated technical implant control, individual settings, upgrades). Central databases may create backups of the current MAP (for example in cases of repair), and document technical data and the hearing performance. Some applications 
described here, are already implemented in the routine, others are currently being developed.

Understanding the possibilities of digitization and their implementation in the context of hearing rehabilitation with hearing implants as well as the recognition of the enormous potential for effective, time-efficient structures is essential in order to use this potential. We as ENT specialists are important protagonists in the healthcare system and beside our high specific expertise we have to meet the requirements of our qualification with regard to digital applications so that we might actively contribute to the success of this process.

\section{Inhaltsverzeichnis}

Zusammenfassung

Abstract

Abkürzungsverzeichnis

Einleitung

Hörstörungen: weltweit ein globales Gesundheitsproblem

Cochlea Implantate

Aktuelle Entwicklungen und Herausforderungen in der CI Versorgung

4.4. Fokus Digitalisierung und Cl-Versorgung

Zusammenfassung

Soziale Medien und Internetpräsenz

Digital unterstütztes Hörscreening

Screening bei Kindern

Screening bei Erwachsenen

Screeningtools - Hörtestungen, Fragebögen und Apps

Digitalisierung in der neuroradiologischen Diagnostik

Planungssoftware OTOPLAN

Digitalisierung in der Audiologie

Digitalisierung und $\mathrm{Cl}$-Operation

Intraoperative Bildgebung
Intraoperative audiologische Messung

Digitalisierung in der postoperativen Phase

Ausweg - Digitalisierung

Was bedeutet "Cl-Nachsorge“?

Herausforderung aus Patientensicht

Herausforderung aus Kliniksicht

Lösungsansätze in der Cl-Nachsorge

Hörtraining in der Cl-Nachsorge

Remote-Care-Netzwerke

Evidenz der telemedizinischen Cl-Nachsorge

Outcome

Perspektiven

Problembeschreibung

Thematische Zielsetzung

Ziele, Rolle und Aufgaben im Projekt

Schlussbemerkungen

Literature

\section{Abkürzungsverzeichnis}

$\begin{array}{ll}\text { AHL } & \text { Asymmetric hearing loss } \\ \text { ASSE } & \text { Auditory Speech Sounds Evaluation } \\ \text { AutoART } & \text { auditory response telemetry } \\ \text { AutoNRT } & \begin{array}{l}\text { automatical neural response telemetry } \\ \text { BERA }\end{array} \\ \text { Brainstem evoked response audiometry } \\ \text { CAP } & \text { Compound Action Potential } \\ \text { CBCT } & \text { Cone Beam CT } \\ \text { CDS } & \text { Clinical decision support } \\ \text { CI } & \text { Cochlea Implantat } \\ \text { CT } & \text { Computer Tomographie } \\ \text { DPOEA } & \text { Distorsionsprodukte Otoakustisch evozierter } \\ & \text { Potentiale } \\ \text { DVT } & \text { Digitale Volumen Tomographie } \\ \text { eABR } & \text { electrical evoked auditory brainstem response } \\ \text { eCAP } & \text { electrical evoked compound action potential } \\ \text { eSRT } & \text { electrical evoked stapedius reflex test }\end{array}$

FOX

$\mathrm{HL}$

HRQoL

iOs

KDD

LMIC

MRT

MVBT

NFS

NRT

PET

PTA

ReHa

SOE

SSD

TEOAE
Fitting to Outcomes eXpert

Hearing loss

Health related quality of life

mobiles Apple-Betriebssystem

Knowledge Discovery in Databases

Low- and Middle Income country

Magnet-Resonanz-Tomographie

Master Volume, Bess, Treble

Nucleus fitting software

Neural Response Telemetry

Positronen-Emissions-Tomographie

Pure Tone Audiometry

Rehabilitation

spread of excitation

Single-sided deafness

Transitorisch evozierte otoakustische Emissionen 


\section{Einleitung}

Die Entwicklungen der letzten Jahre haben zu einem rasanten Aufschwung im gesamten Bereich der Hörimplantate geführt und sind damit in der HNO-Heilkunde ein Bereich mit höchstem Innovationspotential. Neue, bzw. erweiterte Indikationen gingen einher mit Entwicklungen im Bereich der Implantate, neuen OP-Techniken und entsprechend angepassten Rehabilitationsstrategien. Die Vielfalt der Versorgungsmöglichkeiten stellt uns als HNO-Ärzte vor neue, sehr reizvolle Herausforderungen. Um diesen komplexen Prozess zu meistern und zu gestalten, bedarf es neben einer engen interdisziplinären Zusammenarbeit und kontinuierlichen Weiterentwicklung der Therapie auch ganz neuer Strategien.

Die zunehmende Digitalisierung des gesellschaftlichen Lebens verändert auch die Anforderungen an eine moderne Gesundheitsversorgung und bietet zugleich Chancen für ein effizienteres Gesundheitssystem

Die digitale Revolution stellt alle Beteiligten vor große Herausforderungen. Aber sie eröffnet auf der anderen Seite, bspw. im Bereich der Hörrehabilitation, um die es in diesem Referat gehen soll, auch vielfältige Chancen. Das Verständnis über die Möglichkeiten der Digitalisierung und deren Einsatz im Bereich der Hörrehabilitation mit Hörimplantaten und die Erkenntnis über das enorme Potenzial für effektive, zeiteffiziente Strukturen ist unabdingbar, um dieses Potenzial zu nutzen und diesen Prozess aktiv mitzugestalten.

\section{Hörstörungen: weltweit ein globales Gesundheitsproblem}

Über $5 \%$ der Bevölkerung weltweit - oder 466 Mio. Menschen sind nach den aktuellen Angaben der WHO von Hörstörungen betroffen (432 Mio. Erwachsene und 34 Mio. Kinder). Es wird erwartet, dass im Jahre 2050 über 900 Mio. Menschen, das bedeutet jeder Zehnte weltweit von einer Hörstörung betroffen sein wird. (Erwachsene $\mathrm{HL}>40 \mathrm{~dB}$; Kinder $\mathrm{HL}>30 \mathrm{~dB}$, jeweils für das bessere Ohr). Die meisten Betroffenen gibt es in „low- and middle-income countries“.

Eine unbehandelte Beeinträchtigung des Hörvermögens ist mit erheblichen sozioökonomischen Belastungen und Kosten verbunden, laut WHO jährlich 750 Billionen US\$ [1].

Für die Betroffenen bedeutet es häufig auch eine erhebliche Einschränkung in der Kommunikation und in ihren sozialen Interaktionen. Hörstörungen haben einen signifikant negativen Einfluss auf die Lebensqualität und können v. a. bei älteren Patienten Gefühle von Einsamkeit, sozialer Isolation bis zu depressiven Symptomen hervorrufen [2-7].

Die Prävention, Diagnostik und Behandlung von Hörstörungen ist deshalb nicht nur unter dem „cost-effective“ Aspekt von globalem Interesse, sondern auch für die Betroffenen von großem individuellen Nutzen, der sich über die Verbesserung der auditiven Fähigkeiten hinaus auch auf vielfältige psychologische und psychosoziale Bereiche erstreckt und mit einer nachhaltigen Verbesserung der Lebensqualität verbunden ist [8-12].

Für die Hörrehabilitation kommen in Abhängigkeit von der Art und der Ausprägung der Hörstörung verschiedene Therapieoptionen in Frage. Wir sind als HNO-Ärzte in Deutschland, in einem Land mit höchsten Standards der medizinischen Versorgung, in der sehr privilegierten Situation, unsere Patienten nicht nur umfassend zu beraten, sondern ihnen auch alle Möglichkeiten der modernen The- rapie von Hörstörungen anbieten zu können. Das Spektrum reicht von hörverbessernden Operationen über Hörgeräte bis hin zu den verschiedenen Optionen für Hörimplantate.

\section{Cochlea Implantate}

Die Versorgung von hochgradig schwerhörigen oder gehörlosen Patienten mit einer elektronischen Innenohrprothese (Cochlea Implantat, $\mathrm{Cl}$ ) stellt einen enormen Fortschritt in der Behandlung betroffener Menschen dar. Durch diese Maßnahme wird vielen Betroffenen die Möglichkeit zur umfassenden Hör-und Sprachrehabilitation bzw. bei Kindern das Erlernen der Sprache (Habilitation) ermöglicht [13].

Die Erfolgsgeschichte der Cochlea-Implantate, einer auditorischen Neuroprothese, wurde vielmals beschrieben [14]. In der Regel erreichen postlingual ertaubte Patienten ein offenes Sprachverständnis und können telefonieren. Bei Kindern wird bei früher Implantation nach Eintritt der Ertaubung in der Regel eine nahezu normale Sprachentwicklung erreicht [15].

Aufgrund der rasanten technischen Entwicklung in Verbindung mit verbesserten, atraumatischen OP-Methoden und modifizierten Rehabilitationsstrategien und den dadurch erzielten guten Ergebnissen haben sie sich zur Standardtherapie bei sensorischer Taubheit etabliert.

In eigenen Untersuchungen konnten wir den Zugewinn, gemessen am Sprachverstehen und der Lebensqualität (HRQoL), aber auch hinsichtlich der Reduktion der Stress- und Tinnitusbelastung bis zu einer Verbesserung von psychischen Komorbiditäten in unterschiedlichen Altersclustern, zeigen. [5-7, 10-12,16].

Insgesamt darf das Cochlea-Implantat als Prototyp für den Sinnesersatz gelten. Zurzeit sind weltweit ca. 300000 Patienten mit einem Cochlea-Implantat versorgt [17].

\section{Aktuelle Entwicklungen und Herausforderungen in der Cl Versorgung}

Die Versorgung von Patienten mit einer hochgradigen Schwerhörigkeit ist ein komplexer Prozess, der die interdisziplinäre Zusammenarbeit unterschiedlicher Fachrichtungen erfordert. Zudem findet eine kontinuierliche Weiterentwicklung der Therapie statt, sodass der Erwerb und die Weitergabe der fortlaufend aktualisierten Kenntnisse notwendig ist [13].

Die Entwicklungen in den letzten 15 Jahren haben hier zu einem rasanten Aufschwung im gesamten Bereich der Hörimplantate geführt.

Die Komplexität und Variabilität der Versorgungsmöglichkeiten stellt hohe Anforderungen an die fachliche Expertise der Cl versorgenden Klinik.

Grundsätzlich kommen für eine Cl-Versorgung Patienten in Frage, bei denen mit $\mathrm{Cl}$ ein besseres Hören und Sprachverstehen als mit Hörgeräten absehbar zu erreichen ist [13]. Um ein binaurales Hören zu ermöglichen, ist es zudem notwendig für jedes Ohr eine optimale Therapieoption zu finden.

Indikationen sind deshalb heute neben der beidseitigen sensorischen hochgradigen Schwerhörigkeit und Taubheit sowohl bei Kindern als auch bei Erwachsenen, auch Patienten mit einseitiger Taubheit(SSD) und asymmetrischem Hörverlust (AHL) sowie mit Hochtontaubheit. Aus den neuen, bzw. erweiterten $\mathrm{Cl}$-Indikationen resultiert nicht nur eine erhebliche Steigerung der Anzahl möglicher Cl-Kandidaten, es ergeben sich auch vielfältige Variationen, die von der Versor- 
gung mit $2 \mathrm{Cl}^{\prime}$ s, über ein $\mathrm{Cl}$ und Hörgerät, $\mathrm{Cl}$ in Kombination mit anderen Implantaten, wie z. B. Vibrant Soundbridge oder Bonebridge bis zu einem $\mathrm{Cl}$ bei Normakusis der anderen Seite reichen.

Die erweiterten Indikationen in Verbindung mit der Variabilität der Hörsysteme stellen deshalb auch hohe Anforderungen an die Qualifikation und die fachliche Expertise aller in den gesamten Rehabilitationsprozess eingebundenen Beteiligten.

\section{Ältere Patienten}

Ein weiterer Aspekt ist der zunehmende Anteil an älteren Patienten, die heute mit einem $\mathrm{Cl}$ versorgt werden. Während wir an unserer Klinik erst 2006 den ersten Patienten über 70 Jahre mit einem $\mathrm{Cl}$ versorgt haben, beträgt der Anteil heute (eigene Daten von 20122017) unter den erwachsenen $\mathrm{Cl}$ - versorgten Patienten schon $25 \%$. Dieser Trend wird sich fortsetzen.

Nach Erhebungen des statistischen Bundesamtes waren 2011 circa 22 Mio. Einwohner der BRD über 65 Jahre alt [18].Durch den fortschreitenden europaweiten soziodemografischen Wandel steigt der Anteil älterer Menschen in unserer Gesellschaft zukünftig erwartungsgemäß stark an. Hörstörungen zählen mit einer Inzidenz von ca. 2/3 der über 70-Jährigen zu den Volkskrankheiten [2].

Eine Altersgrenze gibt es entsprechend der Leitlinie „CochleaImplantat-Versorgung und zentral-auditorische Implantate“ [19] nicht und auch unsere eigenen Studienergebnisse zeigen, dass die Cl-Versorgung eine sehr erfolgreiche Therapieoption für die Hörrehabilitation von Patienten über 70 und sogar über 80 Jahre sein kann. Ältere Patienten profitieren nicht nur durch eine verbesserte HRQoL sondern auch hinsichtlich ihrer kognitiven Fähigkeiten [5-7, 20].

\section{Patientenversorgungsgrad und Entwicklung}

Trotz der erheblichen jährlichen Zuwachsraten an Cl-versorgten Patienten in Deutschland ist die Bedeutung der notwendigen Beratungen nicht zuletzt durch den weltweit niedrigen Versorgungsgrad der hörgeminderten bzw. nicht adäquat versorgten Patienten hervorzuheben. Nach Schätzungen der WHO liegt die Prävalenz schwerer Hörverluste (61-80 dB) und der Hörminderungen über $80 \mathrm{~dB}$ für $\mathrm{Er}$ wachsene bei etwa $1 \%$ weltweit [21].

Innerhalb Deutschlands beträgt der prozentuale Anteil der mittels Cochlea-Implantat versorgten Patienten in Bezug auf die Gruppe der Patienten mit einer Cl-Indikation noch weit unter 10\% [22-24]. Zurzeit gibt es allein in Deutschland ca. 1 Mio potentielle Kandidaten für ein Cochlea-Implantat, davon sind aktuell ca. 50000 implantiert [15].

Mit gerade einmal $6 \%$ der Patienten mit Cl-Indikation liegt die Versorgungsrate in den USA auf ähnlich niedrigem Niveau [25]. Insgesamt wurden weltweit erst etwa $300000 \mathrm{Cl}$ implantiert [17].

\section{Langzeit Nachsorge}

Die Versorgungsproblematik Bedarf vs. Ressourcen wird bei steigenden Patientenzahlen und weiter abzusehenden Erweiterungen der Cl-Indikationen noch größer werden. Die implantierende Klinik wird weiter die Verantwortung für den gesamten Rehabilitationsprozess des Patienten tragen müssen. Dieser Versorgungsprozess erstreckt sich von der präoperativen Betreuung und Beratung über die Implantation bis hin zur postoperativen Basis- und Folgetherapie und endet mit der lebenslangen Nachsorge [13].
Wandel in der sozialen und beruflichen Struktur der CI Kandidaten/ CI Nutzer

Der Anteil Cl-versorgter Patienten, die sehr gut in den beruflichen und sozialen Alltag integriert sind, steigt auch in Folge der erweiterten Cl-Indikationen (z. B. SSD).

Gerade diese Patienten wünschen sich Terminangebote in der Nachsorge, die besserer mit ihrer beruflichen oder auch familiären Situation vereinbar sind. Im Idealfall sollte sich die Nachsorge problemlos in den Alltag integrieren lassen.

Problematisch sind in diesem Zusammenhang auch weite Wege in Gebieten mit geringer Bevölkerungsdichte und nur wenigen Rehabilitationsstandorten in großem räumlichen Abstand.

\section{Technische Weiterentwicklungen und versorgte Cl-Nutzer}

Die große Bandbreite an Versorgungsmöglichkeiten geht einher mit einem erweiterten Portfolio an Implantaten, Elektroden und Sprachprozessoren. Auch im Bereich der Zubehörtechnik gibt es eine große Bandbreite und vielfältige Innovationen. Der Wunsch seitens der Patienten als auch der Anspruch seitens der Behandler besteht, dass möglichst viele komplexe Hörsituationen immer besser gemeistert werden können. Dazu ist teilweise Zubehörtechnik erforderlich, welche die Bedienung des Cl-Systems komplexer und damit u. a. für ältere Patienten schwieriger macht.

\section{Qualitätsstandards, Qualitätsmanagement und Datenschutz}

Ziel der Bemühungen aller am Rehabilitationsprozess Beteiligten ist die Erhöhung der Qualität der CI-Versorgung. Entsprechend der Leitlinie erfordert die Cl-Versorgung ein interdisziplinäres Team und ein qualitätsgesichertes Konzept, das von der Indikationsstellung bis zur lebenslangen Nachsorge reicht und das in der AWMF-Leitlinie Cochlea-Implantat niedergelegt ist [19].

Ergänzend hierzu listet das Weißbuch CI den Umfang der Maßnahmen zu einer zusätzlichen Qualitätssicherung der Cl-versorgenden Einrichtung auf [13]. Hier wird neben jährlichen Beschreibungen und Erfassung der organisatorischen, strukturellen, diagnostischen und therapeutischen Standard-Prozesse in einem Qualitätsmanagement- System (inkl. Handbuch) u. a. auch der Einschluss dieser Prozesse in ein QM-Zertifizierungsverfahren genannt.

Zusätzlich soll eine kontinuierliche Erhebung der Cl-RegisterDatensätze erfolgen, da eine Qualitätssicherung im Bereich der $\mathrm{Cl}$ Versorgung die Erhebung von Implantat-bezogenen. Daten unter Berücksichtigung der geltenden Datenschutzgesetze zwingend voraussetzt. Auch diese Maßnahmen inclusive der Erfordernisse des Datenschutzes binden zeitliche und personelle Ressourcen.

\section{Globales Problem}

Hörstörungen sind ein globales Gesundheitsproblem. Cochlea-Implantate werden weltweit implantiert, zunehmend auch in „low- and middle-income countries“ (LMICs), wo die Nachsorge unzureichend organisiert ist. Auch hierfür gibt es einen Bedarf an zukunftsfähigen Konzepten, die diesen Bedingungen gerecht werden.

Zusammengefasst stellen die genannten Entwicklungen:

- Versorgung größerer Patientenzahlen bei gleichbleibendem Personalschlüssel

- Versorgung immer älterer Patienten mit erhöhtem Beratungsbedarf und Komorbiditäten 
- Erweiterte Indikationen und Variabilität der Hörsysteme

- Anforderungen an die Qualifikation und Ausbildung

- Veränderte soziale und berufliche Patientenstruktur

- Notwendigkeit der lebenslangen Nachsorge, die nicht für alle Cl-Träger nur in hochspezialisierten Zentren erfolgen muss

- Einhaltung von Qualitätsstandards, Qualitätsmanagement und Datenschutz

bei eher knapper werdenden personellen und finanziellen Ressourcen (Bedarf vs. Ressourcen) eine enorme Herausforderung dar.

Diese Ausgangslage zwingt alle, die an den organisatorischen, strukturellen, diagnostischen und therapeutischen Prozessen beteiligt sind, zu effektiven Innovationen im Rahmen der Hörrehabilitation mit $\mathrm{Cl}$ und anderen Hörimplantaten.

\subsection{Fokus Digitalisierung und $\mathrm{Cl}$-Versorgung}

Ziel der Bemühungen aller am Rehabilitationsprozess Beteiligter ist die Erhöhung der Qualität der Cl-Versorgung bei gleichzeitiger Erhöhung der Effizienz der eingesetzten Mittel.

Hier kommt der Digitalisierung all dieser Prozesse eine Schlüsselrolle zu. Die WHO definiert E-Health als den Einsatz von Informationsund Kommunikationstechnologien (ICT) im Gesundheitswesen, bspw. in der Forschung, Ausbildung, medizinischen Diagnostik oder auch in der Behandlung. Die Möglichkeiten zum Einsatz von ICT sind vielfältig.

Bereits heute informieren sich viele Patienten im Vorfeld eines Arztbesuches, Trend zunehmend. Damit wird deutlich, dass sich immer mehr Patienten eine aktive Selbstbefähigung (Empowerment) wünschen. Das wird ihnen durch Telemedizin sowie durch medizinische Apps ermöglicht.

Möglichkeiten einer vereinfachten Anpassung (MAP Erstellung) ergeben sich z. B. durch eine (Semi-) Automatische MAP-Erstellung (z. B. NFS, FOX, bzw. andere Artificial Intelligence Anwendungen).

Durch die Online-Anbindung (Telemedizin, Remote Care und Apps) rückt eine ortsnahe Versorgung des Cl-Trägers in greifbare Nähe. Telemedizinische Remote Care Konzepte erlauben auch im Bereich der $\mathrm{Cl}$-Versorgung völlig neue Formen der Patientenversorgung mit aktiver Beteiligung des Patienten, wie z. B. automatisierte technische Implantatkontrolle, Selbstprogrammierung und technologische Upgrades. Zentrale Datenbanken können u. a. die aktuelle MAP z. B. im Reparaturfall speichern, technische Daten und die Hörleistung dokumentieren.

\section{Zusammenfassung}

Einige der oben skizzierten Anwendungen sind heute schon Realität, andere erst in der Entwicklung. Datenspeicherung in zentralen Datenspeichern sowie Vernetzung sind heute in vielen Lebensbereichen gängig. Bei der Betreuung der Cl-Patienten sind derartige Lösungen durchaus im Entstehen oder auch schon implementiert, so die Vernetzung der implantierenden Klinik mit der Nachsorgeeinrichtung.

Aufgrund der hohen Anzahl Cl-versorgter Patienten und der durch Leitlinie und Weißbuch standardisierten, klar definierten Prozessstruktur und -Qualität der Cl-Versorgung, werden in diesem Referat die aktuellen Entwicklungen aus dem Blickwinkel einer Cochlea-Implantat (CI)versorgenden Klinik dargestellt und besprochen.

Dieser Versorgungsprozess erstreckt sich über die Phasen der präoperativen Evaluation und Beratung, der Operation (Implantation) bis hin zur postoperativen Basis- und Folgetherapie und endet mit der durch die versorgende Einrichtung gewährleisteten lebenslangen Nachsorge. Angesichts des angesprochenen Versorgungsgrades wird auch der Bereich Informationen und Screening von potentiellen Kandidaten beleuchtet.

Hierbei werden ganz gezielt spezielle Applikationen herausgestellt, die in der Entwicklung des digitalen Fortschritts und digitaler Strukturen im Rahmen der Cl-Versorgung eine besondere Rolle spielen und für das Verständnis der weiteren Entwicklung wichtig sind.

Angesichts der rasanten Veränderungen, wir sprechen von einer digitalen Revolution, kann diese Übersicht nur eine Momentaufnahme in einem sich schnell entwickelnden Bereich sein. Anspruch auf Vollständigkeit kann nicht erhoben werden.

\section{Cochlea-Implantat und Hörscreening unter dem Aspekt der Digitalisierung}

Bei zunehmendem Versorgungsauftrag - wie bereits in der Einleitung dargestellt - durch stattgehabte und weiterhin abzusehende Erweiterungen der ehemals sehr strengen $\mathrm{Cl}$-Indikationen wird die Notwendigkeit von suffizienten Screeningverfahren speziell für $\mathrm{Cl}$ Kandidaten an Bedeutung deutlich gewinnen. Unter dem Ansatz einer flächendeckenden Identifizierung von Cl-Patienten werden aufgrund der Quantität an Daten bei begrenzten Ressourcen digitale Lösungen gefordert.

\section{Soziale Medien und Internetpräsenz}

Unabhängig vom Alter ist der Austausch in sozialen Medien zwischenzeitlich in unserer Gesellschaft fest implementiert. Gerade für Gehörlose bietet dieses Medium neben der reinen Webseite-Suche eine gute Möglichkeit in Kontakt zu treten und Informationen abzurufen. Natürlich hat diesen Trend auch die Industrie längst erkannt und bietet Kanäle und Blogs rund um das Thema Cochlea-Implantat an, denn mit wenigen „Klicks“ wird eine große Anzahl vorausgewählter Interessenten oder „Follower“ erreicht. Sehr professionell aufgebaut erscheinen die Webpräsentationen der 4 großen Cochlea-Implantat-Hersteller [26-29].

Aiello et al. [30] untersuchten den Einfluss sozialer Netzwerke auf das Stresslevel von Eltern mit gehörlos geborenen Kindern mit potentieller Cochlea-Implantat-Versorgung. Die Autoren fanden keinen Unterschied im Stresslevel gemessen an einem Online- Fragebogeninventar der beiden untersuchten Gruppen mit und ohne Zugang zu einem speziellen sozialen Netzwerk für Betroffene. Einer Analyse englischsprachiger Webseiten mit dem Inhalt Hörverlust und deren Behandlung eingeschlossen Cochlea-Implantaten zufolge sind 64\% der angebotenen Seiten kommerziellen Ursprungs [30]. Nur ein geringer Teil dieser Webangebote erfüllte Score-gebundene, vergleichbare Qualitätskriterien.

Interessant ist das mehrsprachige Angebot des Ida Institute [31], einer unabhängigen non-profit Organisation, die die personalisierte Versorgung von Menschen mit Hörstörungen in den Mittelpunkt stellt. Die Ida Community hilft Patienten, deren Beeinträchtigungen besser zu beschreiben und somit aktiv bei der eigenen Versorgung mitzuwirken und soll ebenfalls dem Behandler ein besseres Verständnis der individuellen Hörschädigung Betroffener ermöglichen. Interessanterweise nutzen v. a. ältere Hörgestörte Online-Angebote mehr als normalhörende Gleichaltrige [32]. 
In der Folge werden auch E-Services bis hin zur Onlineerreichbarkeit von Ärzten und Fernbehandlung durch diese gewünscht. Besonderes Gewicht gewinnt dies im Hinblick auf vor-ärztliche Beratung und Screeninguntersuchungen. Bereits aufgrund der Quantität der Anfragen allein und der hierdurch generierten Datenmengen erscheinen digitale Lösungen erstrebenswert. Die Bedeutung der notwendigen Beratungen ist nicht zuletzt durch den weltweit niedrigen Versorgungsgrad der hörgeminderten bzw. nicht adäquat versorgten Patienten hervorzuheben.

\section{Digital unterstütztes Hörscreening}

Um der Patientengruppe, die eine Indikation zu einer CI Versorgung aufweist und der noch weitaus größeren Kandidatengruppe, die wissen möchte ob ein Cl für sie in Frage kommt, zu entsprechen, werden digitale Lösungen gefordert. Primärziel bleibt die flächendeckende Identifizierung von CI Kandidaten, da der Evaluierungsprozess der Implantatkandidaten an Quantität und Komplexität zugenommen hat.

\section{Screening bei Kindern}

Patientengruppen, die in diesen digital unterstützten Evaluierungsprozess aufgenommen werden sollten, sind einerseits die Gruppe der Neugeborenen und Kleinkinder vor dem Spracherwerb und andererseits die davon abzugrenzende große Gruppe der Erwachsenen.

Durch die Einführung des Neugeborenen-Hörscreenings auf einen Beschluss des Gemeinsamen Bundesausschusses vom 01.01.2009 hin wurde ein flächendeckendes Primärscreening in Deutschland etabliert. Ziel dieser Untersuchungen, aber auch des Trackings der kleinen Patienten mit konsekutiven Nachuntersuchungen ist die Vorverlagerung des Diagnosezeitpunktes in das erste Lebensjahr. Bedeutend werden hier ein- oder beidseitige Hörminderung ab $35 \mathrm{~dB}$ im Hauptsprachbereich gewertet. Einhergehend mit dem früheren Erkennen erfolgt auch eine Vorverlagerung des Versorgungszeitpunktes - sei es durch Hörgeräte oder die Rehabilitation durch ein $\mathrm{Cl}$. Nicht zuletzt durch Verbesserungen der Operationstechnik und des perioperativen Settings konnten Ängste der Eltern vor einer frühen Versorgung relativiert werden [33].

Besondere Bedeutung wird die Digitalisierung für das Tracking und für notwendige Nachuntersuchungen aufweisen: hier können medizinische Apps die Eltern erinnern, aber auch auftauchende Fragen beantworten und Sachverhalte klären. Eine weitere Option stellt die telemedizinische Beratung der besorgten Eltern dar.

Zusammenfassend haben die vergangenen Jahre gezeigt, dass Kinder mit Cl-Indikation sehr früh erkannt werden. Die Anzahl der neu zu versorgenden Kinder wird stagnieren, während die Gesamtanzahl der Cl-Patienten in Deutschland weiter ansteigen wird, weil zunehmend mehr ältere Patienten einer Cl-Versorgung zugeführt werden.

\section{Screening bei Erwachsenen}

Erwachsene mit einer plötzlichen ein- und beidseitigen Hörminderung wie z. B. im Rahmen eines Hörsturzgeschehens, einer spezifischen Entzündung oder traumatischen Ursache und die große und stetig wachsende Gruppe der erwachsenen Patienten mit einer progredienten beidseitigen Hörminderung bis Ertaubung im Prozess der Presbyakusis, bedürfen einer sorgsamen und sicheren Abklärung ihrer Höreinschränkung.
Allein in der Untergruppe der unter Altersschwerhörigkeit zu subsummierenden Patienten steigt die Zahl an Cl-Kandidaten, denen durch eine Hörgerätversorgung eine Teilhabe am Alltag nicht mehr adäquat gewährleistet werden kann, stetig.

Üblicherweise werden unzureichend versorgte, funktionell ertaubte Patienten durch den HNO-Facharzt (a) eingewiesen bzw. durch den betreuenden Haus- und Allgemeinarzt (b).

Ein wachsender Markt ist die Beratung der Patienten durch Akustiker und Hörgeräteanbieter und durch Anbieter von Medizinprodukten bis hin zu weltweit aktiven Cl-Firmen (c). Tendenzen der Kooperation zwischen diesen, aber auch die Zusammenarbeit mit den Gruppen a und b zeichnen sich ab.

Ein weiterer wichtiger Anlaufpunkt werden die Krankenkassen (d) der Patienten selber sein: bereits heute werden die Versicherten umworben, bei besonderen Fragestellungen den direkten digitalen Beratungsweg zu wählen, um „smarte Lösungen“ über digitale und sichere Kanäle zu erhalten. Beratungen über Telefon oder App sollen bereits heute in vielen Fällen eine Alternative zu einem persönlichen Arztbesuch darstellen.

Ein weiteres und sicher nicht zu vernachlässigendes Feld ist die Selbstberatung (e) durch konventionelle Medien und insbesondere durch digitale Angebote mit dem Schlagwort der aktiven Selbstbefähigung (Empowerness). Grundlage der digitalen seriösen Quellen sind meist die Gruppen a, d und in besonderem Maße c [26-29].

Es liegt auf der Hand, dass im Hinblick auf sozioökonomische Aspekte die einzelnen Gruppen in ihrer Beratung verschiedene Schwerpunkte legen. Wunsch bleibt hier, dass auch im digitalen Sektor die Beratungen wissenschaftlich fundiert und evidenzbasiert das Wohl des Patienten im Fokus haben.

Auch in Zukunft wird die endgültige Beratung des CI Kandidaten durch die versorgende Klinik bzw. Therapieeinrichtung erfolgen. Bei abzusehenden steigenden Überweisungsraten [34-36] nach Informationsgewinn auf den Wegen a-e erscheint es sinnvoll, die Patienten mit einer Indikation zur operativen Versorgung durch Screening zu identifizieren.

\section{Screeningtools - Hörtestungen, Fragebögen und Apps}

Adäquate Hilfsmittel werden online erstellte bzw. digital versendete Hörtestungen in Kombination mit Fragebögen sein. Beide Screeningtools gibt es bereits heute und werden zunehmend (Gruppen $\mathrm{c}+\mathrm{d}$ ) genutzt. Die Versendung von reinen Tonaudiogrammen (PTA) an die versorgende Klinik scheint dabei keine geeignete Lösung zu sein, da oft Patienten mit einem inadäquaten Sprachverstehen nicht ausreichend erfasst werden. Sinnvoller erscheint die zusätzliche Erfassung der Hörfähigkeit über Sprachtestungen in Störgeräuschen z. B. als Ziffern-Tripel-Test [37]. Dieses Screeningverfahren ermittelt, wie gut man Sprache in lärmender Umgebung verstehen kann.

Eine weitere Option ist die Kombination des PTA mit dem Einsilberverstehen [24]. Die resultierenden Einstufungen korrelieren gut mit den klinischen Erfahrungen umfangreicherer Untersuchungen. Von besonderem Interesse erscheint hier die Arbeit der Arbeitsgruppe um Ulrich Hoppe aus Erlangen [38]: Retrospektiv wurden 318 Ohren von Cl-Kandidaten hinsichtlich ihrer reellen Versorgung ausgewertet. Nach Klassifizierung zur I) Einleitung einer spezialisierten Cl-Vordiagnostik versus II) Fortführung bzw. Optimierung der Hörgerätenutzung wurde die stattgehabte $\mathrm{Cl}$-Versorgung und das postoperative Sprachverstehen weiter ausgewertet. Von den 96 als $\mathrm{Cl}$ - 
Kandidaten klassifizierten Fällen wurden 34 (35\%) nach Komplettierung der präoperativen Diagnostik mit einem Cl versorgt. Von den im Screeningverfahren als Hörgerätkandidat eingestuften Fällen wurden lediglich 4 Pat. (2\%) mit einem $\mathrm{Cl}$ versorgt, sodass die Autoren korrekt von einem hinreichend spezifischen Screeningverfahren sprechen.

Schwer überschaubar ist die Anzahl der verfügbaren Health-Apps für Android und iOS, darunter „Hörtest-Apps“, „Otoscope-Apps“, aber auch „Hörtrainings-Apps“ und andere mehr. In einem Review zur Erfassung der Hörfähigkeit fanden Bright und Pallawela 11 Studien zu 6 unterschiedlichen Apps [39]. Die Autoren der Studie resümieren eine hohe Varianz der Ergebnisse mit schwankender Sensitivität. Die Anwendung von „Hörtest-Apps“ als Ersatz zum Goldstandard der Reintonaudiometrie wird von den Autoren des Reviews in diesem Zusammenhang nicht empfohlen. Das Hauptproblem derartiger Apps sei die fehlende Kalibrierung, wie Le Prell und Mitarbeiter [40] zeigten. Pegelabhängig wurden im Vergleich zu einem kalibrierten Audiometer 5-10 dB höhere Messwerte angegeben. Trotzdem seien diese Apps für orientierende Messungen nützlich, um bspw. okkulte Hörstörungen ins Bewusstsein zu rufen und dem Patienten eine Behandlungsnotwendigkeit aufzuzeigen [32]. Aufgrund der niedrigen Kosten, der Portabilität und leichten Zugänglichkeit können derartige Apps aber durchaus im Screeningbereich sinnvoll sein, so auch in Ländern mit unzureichender medizinischer Breitenversorgung.

Die Genauigkeit von Fragebögen, die bei Erwachsenen erhoben werden korreliert sehr zufriedenstellend mit dem Ergebnis einer komplexen Cl-Vordiagnostik. Es liegt auf der Hand, dass in unserer zeit- und ressourcenverknappten Welt kein $\mathrm{Cl}$-Kandidat online den kompletten Umfang der Charité-Test-Batterie [7, 8, 41] absolvieren möchte - dies wird einzelnen internationalen Zentren vorbehalten bleiben, aber subjektive Fragebögen zum Hörverstehen [35, 42] und Fragebögen nach kognitiven Fähigkeiten $[36,43]$ sind online problemlos zu erheben.

Darüber hinaus könnte passives Monitoring per App aber auch Datenakquise per Wearables in Zukunft Aufschluss geben über das Hörvermögen ohne dass der Nutzer aktive Zeit investieren muss. Bspw. durch Aufzeichnung der Situation, wenn ein Nutzer aus akustischen Gründen Dialogen nicht mehr folgen kann bzw. durch Dokumentation in welchen Hörsituationen ein User die Lautstärke seiner Endgeräte wiederholt anhebt.

Screeningtools - Big Data

Im Hinblick auf die Kompatibilität der aktuellen und kommenden Gerätegenerationen und deren Vernetzungsgrad (Internet of Things) können in der anfallenden Datenflut diese Parameter anhand von Algorithmen dargestellt werden. Bezüglich des Themas Big Data erscheinen für das Cl-Screening 2 der 5 großen „V“ von besonderer Bedeutung: die Probleme des „Volume“, d. h. jeder Nutzer „produziert“ eine enorm große Datenmenge und der „Variety“, d. h. die Vielfalt der gewonnenen Datentypen: beides ist durch herkömmliche Methoden nicht mehr analysierbar.

Als Nebeneffekt können sich die Endgeräte wie Smartphones aufgrund der gewonnenen Daten den individuellen Hörsituationen anpassen. Dies kann durch Techniken zur Signalverbesserung wie Signalkompression und frequenzabhängige Verstärkung erfolgen, um die Signalqualität anzupassen und die Höranstrengung unter un- günstigen Hörbedingungen zu reduzieren. Neben dem Primärgewinn des Users durch die verbesserte Hörsituation werden zusätzliche Daten gewonnen, die das Hörverhalten im Verlauf abbilden und damit dem Nutzer anzeigen können, wann ein Cl-Screening für ihn angezeigt ist. Die Prognose, dass ein smartes Endgerät dann direkt ein $\mathrm{Cl}$ bestellen wird (analog zu smarten Kühlschränken), erscheint in naher Zukunft nicht realistisch.

Attraktiv wäre auch die Gewichtung von Stimmungen der Nutzer: das Auffangen und Dokumentieren (aktiv und passiv) erfolgt bereits heute und wird wirtschaftlich genutzt. Hierbei könnte der Leidensdruck der Cl-Kandidaten bestimmt werden: es bleibt unbefriedigend und unökonomisch, wenn ein Patient durch ein hochspezialisiertes Screeningverfahren als Cl-Kandidat korrekt identifiziert wird, aber dann im versorgenden Zentrum keinen relevanten Behandlungswunsch aufweist.

\section{Screeningtools - Limitierungen und Datenschutz}

Herauszuheben bleibt als Schwachpunkt dieser webbasierten Screeningtestungen noch die Differenzierung zwischen rein sensorineuralen Schwerhörigkeiten und der Hörminderungen, die durch eine Schallleitungskomponente verursacht sind. Für den HNO-Facharzt stellt die Diagnose z. B. von Cerumen obturans und einer Vielzahl anderer Erkrankungen, die zu einer relevanten Schalleitungsschwerhörigkeit führen, eine Kleinigkeit dar. Somit sollen auch erst nach Überprüfung der webbasierten Verdachtsdiagnose, dass ein $\mathrm{Cl}$ indiziert sein könnte, weitere notwendige Schritte wie Bildgebung eingeleitet werden - insbesondere unter strahlenhygienischen und sozioökonomischen Aspekten.

Bezüglich der Sicherheit und des Datenschutzes in Deutschland - geregelt durch das Datenschutzrecht, das Telekommunikationsgesetz und das Telemediengesetz - wird es eine Herausforderung werden, dass der einzelne User stets die volle Kontrolle über seine Daten behalten wird. Dies wird bereits heute im wachsenden Markt der Fitness-Apps, Medizin-Cloudanbieter und Social-Media-Apps mit Gesundheitsbezug deutlich: die bisherigen Entwicklungen im Bereich der Telemedizin und der Apps zeigen, dass die Digitalisierung im Medizinsektor in rasanten Schritten vorangeht.

In diesem Zuge sind über eine App auf dem Smartphone die Patientendaten zwar zu jeder Zeit an jedem Ort immer verfügbar, aber es bleibt fraglich ob der Patient selbst oder sein Hausarzt in der Lage sein werden, diese adäquat zu verwalten bzw. wer diese „big data“Aufgabe übernehmen werden wird.

\section{Screeningtools - Zusammenfassung}

Die digitale Revolution stellt alle Beteiligten wie Patienten, Ärzte oder Wirtschaftsunternehmen vor große Herausforderungen. Aber sie bietet auf der anderen Seite z. B. im Bereich des CI Screenings jedoch auch viele Chancen - sie müssen nur genutzt werden.

\section{Präoperative Evaluierung vor Cochlea- Implantat}

Die präoperative Evaluierung und Diagnostik vor Cochlea-Implantat (Cl)-Operation umfasst die HNO-spezifische Anamnese und klinische Untersuchung, speziell die Ohrmikroskopie. Zudem erfolgt die audiometrische Testung (Tonschwellenaudiometrie, Sprachaudiometrie, Hörgeräte-Überprüfung) und objektive Hörprüfung (Tympano- 
gramm, TEOAE/DPOAE, Klick-BERA). Des Weiteren wird eine Vestibularisprüfung durchgeführt und es erfolgt die neuroradiologische Diagnostik und Befundung durch die Radiologie.

Im Rahmen der Cochlea-Implantat-Diagnostik spielt die Digitalisierung im Bereich der klinischen Untersuchung, der Bildgebung und der Audiologie eine wichtige Rolle.

\section{Digitalisierung in der neuroradiologischen Diagnostik}

Ein Unterpunkt der Digitalisierung in der Cl-Diagnostik ist die Digitalisierung in der neuroradiologischen Diagnostik. Einerseits soll somit eine digitale Verknüpfung von klinischen Patientendaten und -diagnosen und der Bildgebung stattfinden um mithilfe künstlicher Intelligenz Algorithmen zur digitalen Befunderhebung herzustellen. Andererseits kann so eine zentrale Vernetzung der Daten von Kliniken und Praxen stattfinden und mithilfe von „Big Data“ zentral gespeichert werden ( $\triangleright$ Abb. 1). Dies erleichtert den behandelnden Ärzten den Zugriff zu Bilddateien, die in einem anderen Institut bzw. in einer Praxis angefertigt wurden, erleichtert die Diagnosefindung und Weiterbehandlung der Patienten und erspart eine Überbeanspruchung der Kapazitäten in der Radiologie und somit zusätzliche Kosten. Hierdurch kann zudem Strahlenbelastung der Patienten durch unnötige Bildgebung (CT, Röntgen) vermieden werden [44, 45].

Zur Vermeidung von „Überdiagnostik“ wurde in den USA in den frühen 2000ern hierfür ein clinical decision support (CDS) System entwickelt um eine möglichst sinnvolle Anwendung der verschiedenen bildgebenden Verfahren sicherzustellen [46, 47]. Hierbei wenden überweisende Ärzte vor Indikationsstellung von CT, MRT und PET-CT zunächst das CDS System an, welches evidenzbasiert Empfehlungen zur Bildgebung ausspricht. Dieses Verfahren wurde durch Präsident Obama im „Protecting Access to Medicare Act of 2014“ bestätigt und wird aktuell in allen Bundesstaaten der USA angewandt [45]. In Deutschland ist dieses standardisierte Verfahren als Unterstützung für die Entscheidungsfindung des Radiologen noch nicht regelhaft in Gebrauch.

Eine zukünftige Entwicklung im Bereich der Digitalisierung in der bildgebenden Diagnostik ist die individuelle Patientenbetreuung und Erstellung von Befunden basierend auf einer Echtzeit Analyse der radiologischen Aufnahmen durch Big Data Techniken. Hierbei werden Patientendaten und Bilddaten mit Daten aus klinischen Studien, aus medizinischen Fachzeitschriften und medizinischen Datenbanken korreliert und ausgewertet [47].

Bei der Cl-Diagnostik kann eine solche Big Data Korrelation der radiologischen Bildgebung v. a. bei komplexen Innen -und Mittelohrfehlbildungen aufschlussreiche Vergleichsbefunde aufzeigen. Bei der Planung der $\mathrm{Cl}$-Operation kann somit auf die individuellen anatomischen Gegebenheiten des Patienten Rücksicht genommen werden.

\section{Planungssoftware OTOPLAN}

Eine Neuheit im Bereich der präoperativen Bildgebung im Cl-Bereich ist die Software OTOPLAN. Hiermit können neuroradiologische Bilder (CT, MRT, DVT) mit einer neuen Tablet-basierten Planungssoftware ausgewertet werden, die Cochlea kann exakt vermessen werden und dadurch die Elektrodenlänge des $\mathrm{Cl}$ individuell auf den Patienten abgestimmt werden. Die Software OTOPLAN wurde durch die Firmen MedEl und Cascination entwickelt ( $\triangleright$ Abb. 2). Diese Planungssoftware dient dazu 3D Rekonstruktionen des Felsenbeins mittels Com- putertomografie bzw. Cone Beam-CT (CBCT) zu generieren. Diese sind auf die individuelle Anatomie des Patienten abgestimmt und ermöglichen so eine präzise präoperative Darstellung des OP-Gebietes und gefährdeter Strukturen wie des N. facialis.

Eine weitere innovative Anwendung dieser Software liegt bei der Vermessung der Cochlea und somit der Planung der optimalen $\mathrm{Cl}$ Elektrodenlänge. Eine solche personalisierte Cl-OP-Planung führt zu einer möglichst atraumatischen Implantation und lässt auf eine postoperative Verbesserung der Hörrehabilitation hoffen. Die Planungssoftware wurde erstmals von Gerber et al. [48] in einer experimentellen Studie an Kadavern zur Roboter-gestützten minimal invasiven Hörimplantat-Operation eingesetzt. Hierbei konnte die Operation in allen Fällen erfolgreich mit der Software geplant werden. Eine weitere Studie von Ping et al. [49] bestätigt die genannten positiven $\mathrm{Er}$ gebnisse des Planungs-Tools. Mithilfe der Software wurde der N. facialis in vorhandenen CBCT-Bildern in seinem Verlauf im Felsenbein hervorgehoben,um ihn intraoperativ zu schonen.

Weitere Studien zur klinischen Anwendung der Software OTOPLAN stehen bisher noch aus.

\section{Digitalisierung in der Audiologie}

Nicht nur im radiologischen, sondern auch im audiologischen Bereich ist eine zentrale Vernetzung der Patientendaten sinnvoll und möglich wie Mellor et al. [50] in ihrer Publikation beschreiben. „Data Mining “ beschreibt den Prozess, wie Wissen aus großen Datenbeständen extrahiert und in sinnvollen Zusammenhang gestellt wird. Hiermit können Daten v. a. im Bereich der Forschung schneller gefiltert und ausgewertet werden. Dies wird zusammengefasst als KDD (Knowledge Discovery in Databases) Prozess, der in der $>$ Abb. 1 dargestellt wird.

Im audiologischen Feld ist eine Data-Mining-Software ebenfalls sinnvoll und wurde erstmals von Cox et al. [51] (8) bei der International Conference on Computational Intelligence 2004 vorgestellt. Aus 180000 individuellen Audiologie-Befunden von 23000 Patienten wurden heterogene Daten wie Audiogramme, demografische Daten und Texte verwendet und mittels statistischen und neuralen Techniken ausgewertet. Dieses Projekt war Teil der nationalen „Modernising Hearing Aid Services“ Initiative des UK. Zukünftig wäre eine solche nationale Initiative auch im Cochlea-Implantat-Bereich erstrebenswert.

Ein weiterer wichtiger Punkt im Bereich der Audiologie ist die Zentralisierung von Daten, die in England und in Skandinavien in sehr vielen Kliniken und HNO-Praxen durch die Datenbank Audit-Base der Firma Auditdata (auditdata.com) realisiert wird. Diese Datenbank enthält Informationen bezüglich des Patienten, der Audiometrie, des Implantats bei Cl-Patienten, des Hörgerätes bei Hörgeräte-Patienten sowie bezüglich der Hörrehabilitation.

Als Ausblick für die Cl-Patienten ist eine Vernetzung audiologischer Daten sowie eine einheitliche Datenbank möglich und zukünftig realisierbar. Die Audiometrie-Daten können so einheitlich gespeichert werden und sowohl in der Sprechstunde, im OP, als auch in der HNO-Praxis eingesehen werden.

Daten zum Implantat können ebenfalls in der Datenbank gespeichert werden und die erhobenen Daten an die Rehabilitations-Einrichtung weitergeschickt werden. Somit wäre auch das Feedback aus der Rehabilitations-Einrichtung an die Operateure und Ärzte in der Klinik und der Praxis vereinfacht möglich. 


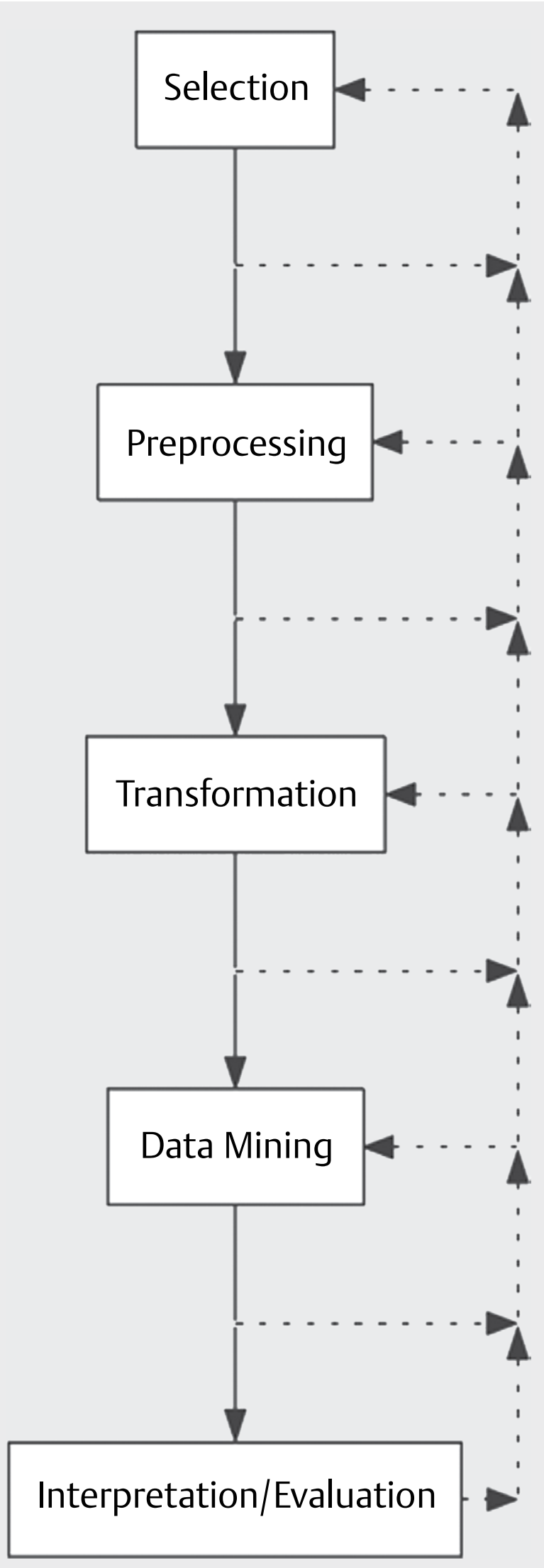

- Abb. 1 Big data in Audiology. Selection=Datenselektion, Preprocessing = Datenbereinigung, Transformation = Datenübertragung in eine nützliche Form, Data Mining = Datenklassifizierung und -gruppierung, Interpretation= Dateninterpretation. Quelle: [50].
Eine weitere zukunftsträchtige Entwicklung ist eine Cochlea-Implantat-Diagnostik App, in Anlehnung an die bereits bestehende App „iHealth“. Hierbei kann der Patient alle bei der Diagnostik erhobenen Daten (Klinischer Befund, Audiometrie, Hörnervtest/PromTest, Vestibularis-Prüfung, CT, MRT) speichern. Dieses Thema wird im Unterpunkt „Hörrehabilitation“ näher beleuchtet.

\section{Operation und stationärer Aufenthalt unter dem Aspekt der Digitalisierung \\ Digitalisierung und $\mathrm{Cl}$-Operation}

Ein Ziel der intraoperativen Digitalisierung ist die dynamische Vernetzung verschiedener Komponenten im OP-Saal, dieses Thema wird an anderer Stelle in diesem Referateband ausführlich behandelt. In diesem Abschnitt soll speziell die intraoperative Digitalisierung bei Cochlea-Implantat-Operation beleuchtet werden.

Hierbei ist die dynamische Vernetzung computergesteuerter Geräte und invasiver Geräte wie Bohrer und Schneideinstrumente besonders wichtig. Diese Vernetzung dient zur Fehlervermeidung und Optimierung des Ablaufs. Des Weiteren wird hiermit die Effizienz der Abläufe verbessert und die Operationszeit verkürzt. Damit werden Ressourcen eingespart und die Patientensicherheit erhöht.

Im OP soll zudem die „Interoperabilität“ gefördert werden, das heißt der Operateur und sein Team sowie der Anästhesist und sein Team können an den Monitoren dieselben Patientendaten zentralisiert und gebündelt abrufen. Weitere für den Operateur relevante Innovationen sind die sterile Steuerbarkeit von verschiedenen Geräten wie bspw. Navigation und OP-Tisch, die an einem zentralen Monitor angezeigt werden. Eine weitere hilfreiche Neuigkeit ist die Vernetzung von Bildmaterial wie CT und MRT mit dem OP-Mikroskop, sodass am Monitor des Mikroskops die präoperative Bildgebung angesehen werden kann [52-54].

Die zentrale digitale Patientenakte, in der sowohl chirurgische Daten, anästhesiologische Daten als auch die präoperative Diagnostik umfasst, erleichtert die spätere Erstellung von Berichten und die Auswertung der Daten hinsichtlich Komplikationen und Fehlervermeidung.

Diese Vernetzung im OP wird an der Universität Leipzig im Innovation Center Computer Assisted Surgery (ICCAS) in einem ModellOP erprobt und erforscht. Zuvor wurde im Projekt OR.NET an der Universität Aachen dasselbe Ziel verfolgt [52-54]. Die Projekte werden bzw. wurden vom Bundesministerium für Bildung und Forschung unterstützt.

Eine Neuheit im Bereich der intraoperativen Digitalisierung im ClBereich ist die Roboter-gesteuerte Cl-Operation, die 2009 von Klenzner et al. [55] beschrieben wurde und später auch von Caversaccio et al. [56] in einer klinischen Studie vorgestellt wurde. Es wurde ein System für die Roboter-gesteuerte Cochlea-Implantat Operation entwickelt und an einem Patienten erfolgreich angewandt. Bei der Operation wird der Zugang zur Cochlea über die posteriore Tympanotomie von diesem Roboter-System gebohrt. Diese Methode ist noch zeitintensiv mit einer OP-Dauer von etwa 3 Stunden [56], sie birgt allerdings das Potenzial in Zukunft auch mit kürzerer OP-Dauer durchgeführt zu werden. Zudem konnte in der Studie die Effektivität, Sicherheit und Durchführbarkeit dieser Methode herausgestellt werden. Vorteile dieser Methode können in Zukunft eine individuelle Planung des Zugangswegs zur Cochlea sein, sowie eine schonendere Elektrodeninsertion und eine exakte Positionierung der Elektrode in der Cochlea. 


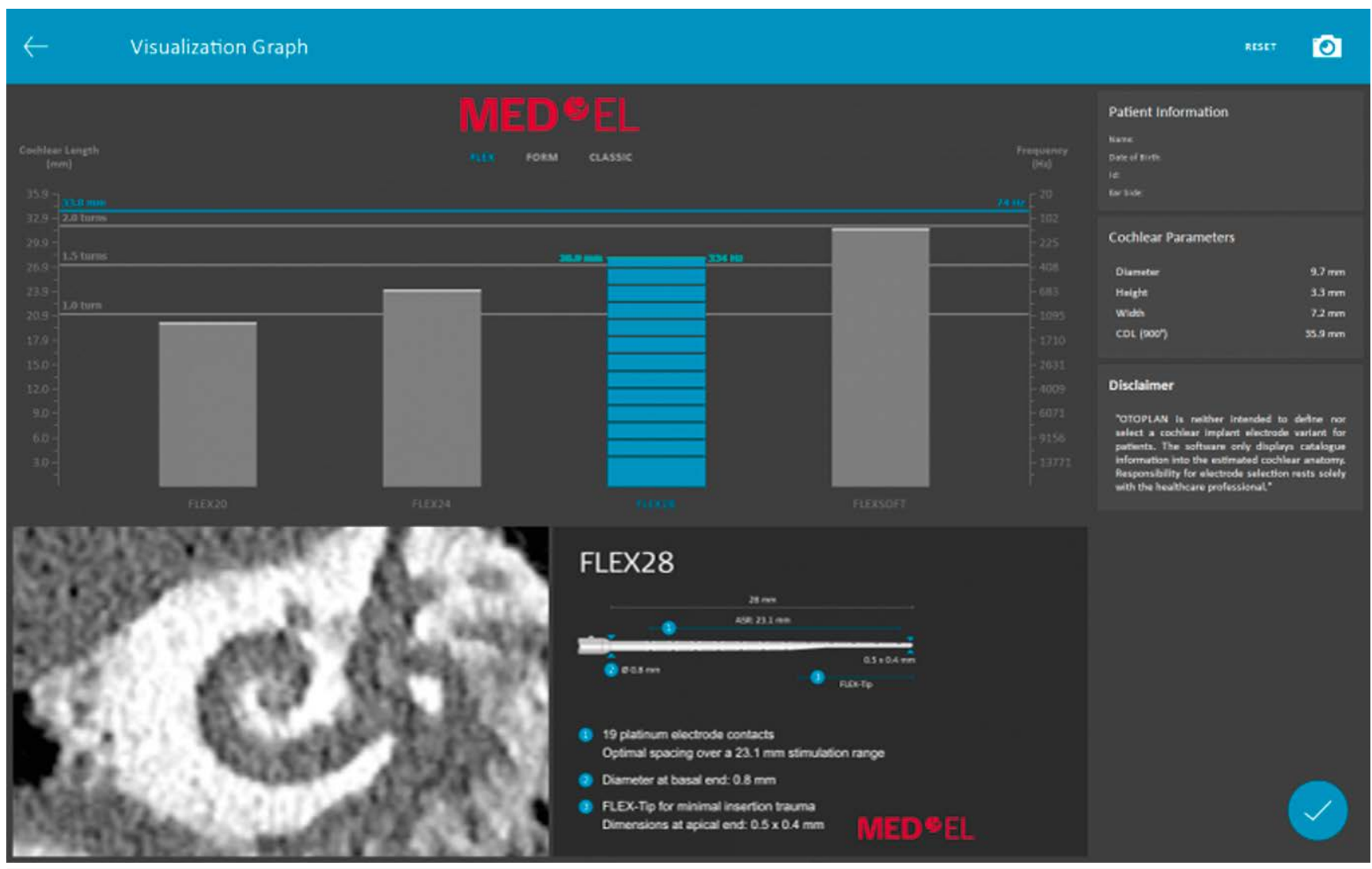

Abb. 2 OTOPLAN-Software. Quelle: https://blog.medel.pro/otoplan-future-otological-surgery. Mit freundlicher Genehmigung von MED-EL.

\section{Intraoperative Bildgebung}

Die intraoperative Bildgebung bei Cl-OPs ist eine hilfreiche Innovation v. a. bei schwierigen anatomischen Verhältnissen und Innenohrfehlbildungen. Cosetti et al. [57] berichten über die intraoperative Bildgebung mittels Röntgenaufnahme nach Stenvers. In wenigen Fällen konnte durch die intraoperative Bildgebung ein Tip-Rollover oder die extracochleäre Lage der Elektrode detektiert und mittels Backup Device sofort korrigiert werden. Vittaro et al. [58] beschreiben in ihrer Studie ebenfalls die Detektion einer Elektrodenfehllage mittels intraoperativer Röntgenaufnahme. Die intraoperative Röntgenaufnahme wird in vielen Kliniken zunehmend verdrängt durch die C-Arm Fluoroskopie [59, 60], welche bei Cl-Operationen nach Elektrodeninsertion 3D Röntgenaufnahmen der Elektrodenlage mit geringer Strahlenbelastung anfertigen kann. Zudem wird auch das Cone Beam-CT bzw. die Digitale Volumen Tomografie (DVT) mit Bildverstärkern oder Flachpanel-Detektoren zunehmend intraoperativ und postoperativ zur bildgebenden Lagekontrolle der Cl-Elektrode angewandt [61]. Die intraoperative CT-Bildgebung wurde in bisherigen Studien v. a. bei Innenohrfehlbildungen und anatomischen Besonderheiten wie einem aberranten Verlauf des N. fazialis in der Literatur eingesetzt [62-64]. Yuan et al. [63] beschreiben in einer Fallserie die intraoperative CT-Bildgebung bei 10 Patienten. Die Elektrodenfehllage bei 2 Patienten konnte mittels CT-Scan detektiert werden und während der OP korrigiert werden. Stelter et al. [64] berichten über die erfolgreiche intraoperative CT-Bildgebung und Anwendung der Navigation mittels BrainLAB bei der Cl-Elektrodenin- sertion bei einem Patienten mit posttraumatischem sensorineuralem Hörverlust.

Während die postoperative Bildgebung zur Lagekontrolle der Elektrode den Goldstandard darstellt, ist die intraoperative Bildgebung bei $\mathrm{Cl}$-OPs zwar wünschenswert, allerdings wird sie aufgrund der Kostenintensität und eingeschränkter Anwendbarkeit bspw. eines DVT im OP-Saal und somit unzureichender Auslastung des Gerätes im OP auch in Zukunft in vielen Kliniken nicht verfügbar sein. Dementsprechend ist es erstrebenswert mithilfe audiologischer Messmethoden eine Elektrodenfehllage zu diagnostizieren um nicht auf eine intraoperative Bildgebung angewiesen zu sein.

\section{Intraoperative audiologische Messung}

Die audiologische Qualitätskontrolle während der Cochlea-Implantat-Operation erfolgt mittels 3 Messverfahren, die in der Regel durch einen Audiologen im OP durchgeführt werden:

Mittels Telemetrie werden die Impedanzen der intracochleären Elektroden bestimmt. Zudem wird das Summenaktionspotential des Hörnerven gemessen (electrical evoked compound action potential, eCAP) um die Antwort des Hörnerven auf elektrische Stimulation festzustellen. Der Stapediusreflex wird durch elektrische Stimulation geprüft (electrical evoked stapedius reflex test, eSRT).

Diese Messverfahren können auch ferntelemetrisch durchgeführt werden, d. h. der Audiologe befindet sich nicht im OP-Saal, sondern an einem Arbeitsplatz mit Computer und mit Verbindung zum OP (Webcam und Lautsprecher). Ein solches Vorgehen wurde erstmals 
2008 von Shapiro et al. [65] beschrieben. Yanov et al. [66] haben dieses Verfahren in einer aktuellen prospektiven randomisierten Studie mit dem klassischen Verfahren verglichen. Bei der ferntelemetrischen Messung wurde zusätzlich zur Standard-Ausrüstung jeweils ein Computer mit Netzwerk-Verbindung im OP und am Arbeitsplatz des Audiologen benötigt sowie ein visuell-auditorisches System (Webcam und Lautsprecher). Es konnte eine korrekte Messung durch die Remote Network Connection festgestellt werden, die sich nicht signifikant von der klassischen Messmethode im OP unterschied. Zudem wurde eine signifikante Zeitersparnis durch das ferntelemetrische Verfahren gemessen (10,04 vs. 18,64 min).

Die Messung der ECAP kann auch automatisch als automatical neural response telemetry (AutoNRT) bzw. AutoART (auditory response telemetry) durchgeführt werden. Die Technologie der AutoNRT wurde erstmals von Botros et al. [67] beschrieben und van Dijk et al. [68] berichteten über die klinische Anwendbarkeit. Das Nucleus Cochlear Implant System (Cochlear Limited, Australien) bzw. das Maestro System (MED-El, Innsbruck, Österreich) können mit einem Algorithmus, der auf einer Machine Learning Technik und Entscheidungsbaum-Analyse beruht, automatisch den Schwellenwert, der eine Reizantwort des Hörnerven auslöst, bestimmen [69]. Tavartkiladze et al. berichten über zuverlässige Ergebnisse mit der Auto-NRT Technologie im Vergleich zur manuellen NRT-Messung. Zudem ist die Auto-NRT Messung zeitlich signifikant kürzer. Ein negativer Aspekt dieser Messmethode ist die eingeschränkte Anwendbarkeit, denn nicht bei allen Patienten ist diese Messmethode möglich. Die Pulsbreite kann bei der Auto-NRT Messung nicht verändert werden und somit muss bei ausbleibender Reizantwort die Messung manuell durchgeführt werden und die Pulsbreite ggf. verlängert werden.

Zur Detektion einer Elektrodenfehllage haben Grolman et al. [70] intraoperativ die Ausbreitung der neuralen Erregung (engl. spread of excitation, SOE) gemessen. In der Studie wurde die Messung sowie eine intraoperative DVT bei $72 \mathrm{Cl}$-Operationen durchgeführt. In 4 Fällen trat eine Elektrodenfehllage auf, die der erfahrene Audiologe anhand der SOE identifizieren konnte und die der Audiologe nach Elektrodennummer identifizieren konnte. Zusammenfassend konnten mithilfe der Bildgebung und der SOE Messung wertvolle Informationen hinsichtlich der Elektrodenlage in der Cochlea gewonnen werden.

Aufgrund der erweiterten Indikationsstellung der Cochlea-Implantat-Versorgung werden auch Patienten mit Vestibularisschwannom (VS) und Ertaubung mittels CI hörrehabilitiert. In diesem Zusammenhang ist eine intraoperative Hörnervtestung nach VS-Resektion sinnvoll um zu entscheiden, ob eine $\mathrm{Cl}$-Indikation besteht. Die Firma MEDEl hat hierfür eine Cochlea-Testelektrode entwickelt, die für die intraoperative Messung der Funktion des Hörnerven konzipiert wurde. Wenn bei einer Tumorresektion die Erhaltung des Hörnerven fraglich ist, so kann die „Cochlear Test Electrode“ ( $\mathbf{A b b . ~ 3 ) ~}$ wie eine $\mathrm{Cl}$-Elektrode in die Cochlea eingeführt werden und es erfolgt die intracochleäre Stimulation des Hörnerven. Bei intaktem Hörnerven können electrical evoked auditory brainstem response (eABR) Antworten registriert werden. Für diese Messung ist die Cochlea-Testelektrode notwendig ( $\triangleright$ Abb. 3), ein ABI Verbindungskabel, eine $A B I$ Stimulator Box, ein EABR Messgerät und EEG Elektroden ( Abb. 4).

Lassaletta et al. [71] berichten in ihrer Studie über die Anwendung der intracochleären Testelektrode bei $10 \mathrm{Cl}$-Patienten. Nach Identifikation des runden Fensters wurde intraoperativ zunächst die Testelektrode eingeführt um eABR Antworten abzuleiten, anschließend wurde diese entfernt, das $\mathrm{Cl}$ eingesetzt und erneut eABR Antworten gemessen. Die Ergebnisse der Testelektrode und des CI wurden verglichen und ergaben keine signifikanten Unterschiede hinsichtlich Latenzen und Amplituden. Cinar et al. (30) beschreiben in ihrer Studie die Anwendung der intracochleären Testelektrode bei Patienten
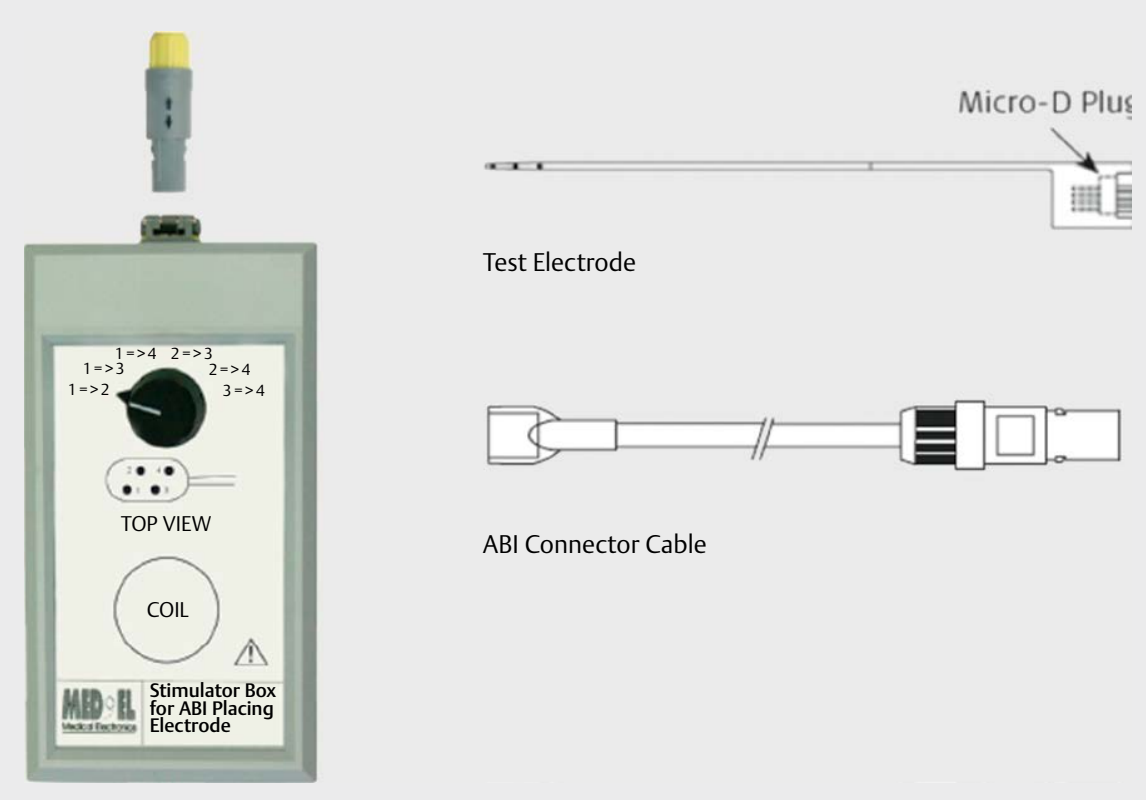

Test Electrode

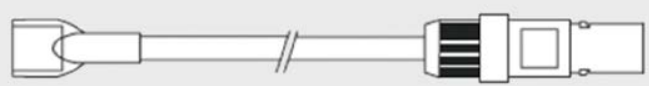

ABI Connector Cable

ABI Stimulator Box and Test Device

Abb. 3 Cochlear Test Electrode (mit freundlicher Genehmigung von MED-EL). 
mit Innenohr-Fehlbildungen. Die Testelektrode wurde angewandt um anhand der abgeleiteten eABR eine Entscheidung für ein Cochlea-Implantat bzw. für ein Hirnstammimplantat (ABI) zu treffen. Es konnte zudem festgestellt werden, dass mit zunehmendem Grad der Fehlbildung die positiven eABR Messungen ausblieben.

Zusammenfassend sollte die cochleäre Testelektrode bei Vestibularisschwannom Resektionen mit unklarem Hörnervenstatus Anwendung finden, da positive eABR Messungen auf eine erfolgreiche Hörrehabilitation mit Cochlea-Implantat hinweisen. Weitere Studien zur Cochlear Test Electrode stehen bisher aus.

\section{Digitalisierung im Rahmen der Cochlea- Implantat-Rehabilitation}

\section{Digitalisierung in der postoperativen Phase}

Nach erfolgreicher Cochlea-Implantation folgt im weitesten Sinne die Phase der Nachsorge, die nach [13] in 3 Abschnitte unterteilt werden kann, die Basistherapie (Tag 1 postoperativ bis 6 Wochen postoperativ), die Folgetherapie (ca. 6 Wochen bis 1 Jahr (bei Kindern 3 Jahre) postoperativ) und schließlich die lebenslange Nachsorge ( $\triangleright$ Abb. 5) mit in der Regel einjährigen Abständen der Nachsorgetermine.

\section{Basistherapie}

Nach initialer Heilungsphase erfolgt die Erstanpassung des Soundprozessors. Der Zeitpunkt wird je nach $\mathrm{Cl}$-Zentrum unterschiedlich gewählt und liegt in der Regel im Bereich der ersten 6 Wochen postoperativ. Zuweilen wird eine erste „Probestimulation“ am ersten oder zweiten postoperativen Tag vorgenommen, in der Regel aber die erste Stimulation und erste Anpassung des Sprachprozessors in ein und derselben Sitzung.

Dieser erste „Hörtermin“ mit dem Cochlea-Implantat stellt für die frisch implantierten Patienten eine extrem emotionsbeladene und anspruchsvolle Situation dar. Sie wissen nicht genau, was auf sie zukommt. Nicht-rationale Ängste und Sorgen und eine starke Aufregung sind häufig anzutreffen.

So „aufgeladen“ kommen die Patienten in die Erstanpassung. Von hier soll der Patient mit einem möglichst erfolgversprechend angepassten Sprachprozessor entlassen werden, das allein erfordert ein hohes Maß an Konzentration und Mitarbeit durch den Patienten. Darüber hinaus erhält er aber auch eine Menge an Informationen zur Handhabung der ihm nun übergebenen Technik einschließlich umfangreichem Zubehör.

Wie kann diese Herausforderung am besten beherrscht werden? Eine Möglichkeit bestünde in der sowohl für die implantierende Klinik als auch den Patienten sehr zeitaufwendigen Aufteilung der Erstanpassung in 2 oder mehr Termine. Eine andere, zeit- und kostensparende Möglichkeit besteht in der Reduzierung der für das Sprachprozessorfitting erforderlichen Zeit und Schonung der mentalen Kapazität des Patienten durch auf objektiven Daten basierende Anpassprozesse bei Nutzung von Standardparametern. Hier hat sich ein Fitting mit Unterstützung durch die intraoperativ gewonnenen Compound Action Potentials (CAP) sowohl bei Kindern als auch bei Erwachsenen bewährt. Smoorenburg et al. [73] konnten an 27 erwachsenen $\mathrm{Cl} 24 \mathrm{M}$-Trägern eine signifikante Korrelation zwischen der Steilheit der psychophysischen und der NRT-Schwellen-Kurve

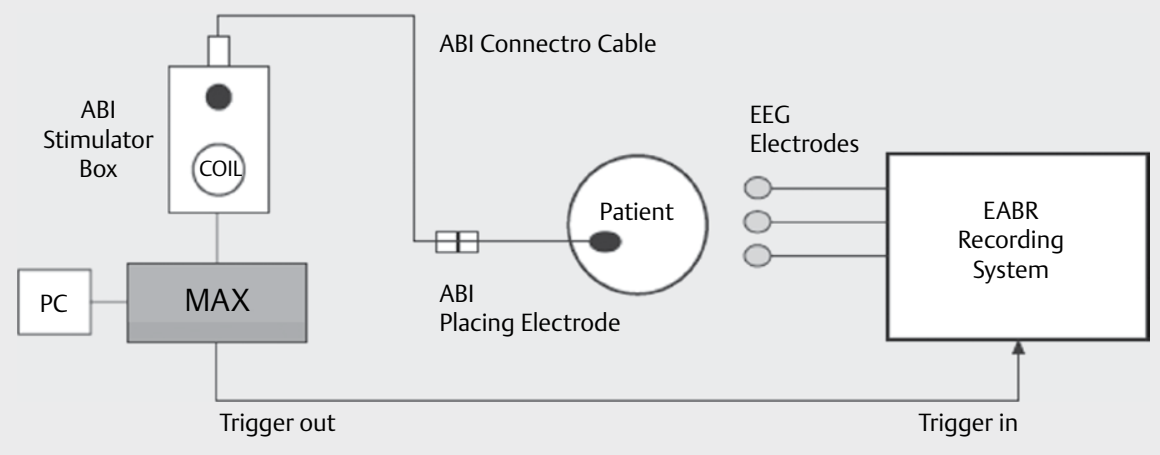

Abb. 4 Setup der eABR Messung mittels Cochlear Test Electrode (mit freundlicher Genehmigung von MED-EL).
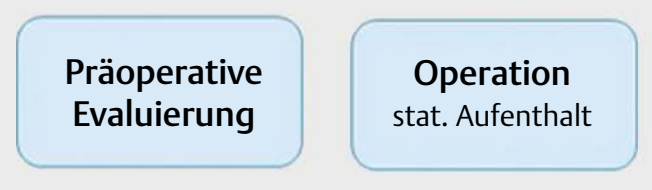

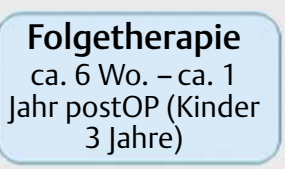

Nachsorge

$>1$ (bei Kindern > 3)

Jahre postOP

\section{Cl-Versorgung}

Abb. 5 zeitlicher Verlauf der Cl-Versorgung 
$(r=0,82)$ zeigen. Das aufwendige Procedere der individuellen Bestimmung von kanalspezifischen Threshold- und Comfort-Leveln kann ggf. in späteren Sitzungen zur Verfeinerung der Anpassung erfolgen.

\section{Folgetherapie}

Der Basistherapie folgt die bei Erwachsenen etwa ein Jahr, bei Kindern etwa 3 Jahre dauernde Folgetherapie, die in audiologische und hörtherapeutische Folgetherapie unterteilt werden kann [13].

Ziel der Bemühungen aller im Rehabilitationsprozess Beteiligten ist die Erhöhung der Qualität der Versorgung bei gleichzeitiger Erhöhung der Effizienz der eingesetzten Mittel durch Automatisierung und Standardisierung. Wie schon in der Einleitung ausgeführt, stellen steigende Patientenzahlen bei eher knapper werdenden personellen und finanziellen Ressourcen eine enorme Herausforderung dar. Diese Ausgangslage zwingt zu effektiven Innovationen bei der längerfristigen Cl-Nachsorge. Hier kommt der Digitalisierung aller Prozesse eine Schlüsselrolle zu.

Einige, teilweise bereits in der Einführung erwähnte Herausforderungen, die auf die Betroffenen (Kliniken, ReHa-Einrichtungen, Patienten, Angehörige) zukommen, seien hier stellvertretend nochmals benannt:

- Versorgung größerer Patientenzahlen bei gleichbleibendem Personalschlüssel

- Versorgung immer älterer Patienten, für die der häufige Weg zur ReHa-Einrichtung ein Problem darstellt

- Diskrepanz zwischen Terminangeboten in der ReHa und Terminwünschen der Patienten infolge beruflicher Zwänge seitens der Patienten

- Rückläufige Möglichkeit/Bereitschaft der Patienten zur Wahrnehmung häufiger ReHa-Termine infolge starker beruflicher/familiärer Belastung

- Schwierigkeit bei der Wahrnehmung von Nachsorgeterminen aufgrund weiter Wege in Gebieten mit geringer Bevölkerungsdichte und nur wenigen ReHa-Standorten in großem räumlichen Abstand

Der Wunsch seitens der Patienten als auch der Anspruch seitens der Kliniken besteht, dass möglichst viele komplexe Hörsituationen immer besser gemeistert werden können. Dazu ist teilweise Zubehörtechnik erforderlich, welche die Bedienung des Cl-Systems komplexer und damit für ältere Patienten schwieriger macht.

Cochlea-Implantate werden zunehmend auch in „low- and middle-income countries“ (LMICs) eingesetzt, wo die Nachsorge unzureichend organisiert ist.

\section{Ausweg - Digitalisierung}

Daraus folgt zwangsläufig die Notwendigkeit zur Optimierung der mit der Cl-Nachsorge verbundenen Prozesse.

Auf folgenden Gebieten sind derzeit Anwendungen aus dem Bereich der Digitalisierung bereits im Einsatz bzw. aus heutiger Sicht denkbar:

\section{Digitalisierung in der audiologischen Folgetherapie}

Der Einsatz objektiver Messverfahren zur Erfassung individueller Varianzen, so die Verwendung automatisch oder manuell gewonnener Compound Action Potentials (CAP) für die manuelle Anpassung [73], kann zu einer zeitlichen Verkürzung der einzelnen Anpassung führen, wobei das Fitting ggf. sogar durch weniger erfahrenes/qualifiziertes Personal durchgeführt werden kann.

(Semi-)Automatische Anpassverfahren auf der Basis patientenindividueller Daten im Zusammenspiel mit Expertenwissen aus großen Patientenkohorten (BigData) finden immer verbreiteteren Einsatz.

Hier gibt es inzwischen bspw. umfangreiche Erfahrungen mit dem computergesteuerten Anpassassistenten FOX (Fitting to Outcomes eXpert). Künstliche Intelligenz soll ein schnelleres, konsistenteres Anpassen von Cochlea-Implantaten und damit eine bessere Hörqualität ermöglichen.

FOX funktioniert sehr ähnlich einem Navigationssystem im Straßenverkehr, das den Start- und den gewünschten Zielort kennt, über ein immenses Wissen bezüglich der möglichen Wege verfügt und schließlich aus einer Vielzahl der möglichen Routen die z. B. hinsichtlich Wegstrecke oder Fahrtdauer effektivste Strecke herausfindet.

FOX nutzt audiologische Daten wie Hörschwelle, Phonemdiskrimination, Sprachaudiogramm (bspw. ermittelt durch Auditory Speech Sounds Evaluation (ASSE- Fa. Otoconsult)) und Lautheitsskalierung als Grundlage für einen Algorithmus zur Optimierung der Implantat-Anpassung. Die audiologischen Testergebnisse stellen den jeweiligen Ausgangspunkt für die automatische Optimierung dar.

FOX bietet die Möglichkeit, die Testergebnisse und ältere MAPs des Patienten im Vergleich mit anderen, anonymisierten MAPs zu analysieren, um dem Patienten die bestmögliche MAP empfehlen zu können. Dadurch wird der Anpassprozess beschleunigt und die Messergebnisse kommen dem Ideal noch näher. Durch die Aufnahme neuer MAPs und Leistungsdaten in die Datenbank werden die prädiktiven Fähigkeiten von FOX permanent verbessert.

Battmer et al. [74] haben in einer Multizenterstudie mit 27 postlingual ertaubten erwachsenen Patienten mit HiRes90K ${ }^{\mathrm{TM}}$ der Fa. Advanced Bionics untersucht, wie sich Effizienz und Dauer der Anpassung mit FOX und einer konventionellen Anpassmethode unterschieden. Sie konnten zeigen, dass in den ersten 2 Wochen nach Erstanpassung die benötigte Zeit für eine FOX-Anpassung signifikant unter der einer konventionellen Anpassung lag, über die weitere Periode des ersten halben Jahres war der Zeitaufwand beider Verfahren gleich. Es wurde eine Reduktion der Variabilität der Anpassergebnisse zwischen unterschiedlichen Zentren durch die Nutzung von FOX beschrieben.

Meeuws et al. [75] konnten in einer retrospektiven Studie die Lernkapazität des FOX-Algorithmus an 25 postlingual ertaubten erwachsenen Patienten (14 mit einem Implantat der Fa. Cochlear versorgt, 11 mit Advanced Bionics) mit einer mittleren Cl-Nutzungsdauer von 10 Jahren zeigen. Das Sprachverstehen konnte mittels FOX-Programmierung gegenüber der „eigenen“ MAP gesteigert werden.

Auch Vaerenberg et al. [76] konnten den Nutzen von Fox an 8 neu $\mathrm{Cl}$-versorgten, postlingual ertaubten erwachsenen Patienten mit HiRes90K ${ }^{\mathrm{TM}}$ ( Fa. Advanced Bionics) darstellen. Sie sehen den Vorteil des deterministischen Verfahrens in einer Systematisierung der $\mathrm{Cl}$ Programmierung, Reduktion der Fitting-Dauer und einer Optimierung der Hörergebnisse.

Vereinfachte Anpassverfahren auf Basis der CAP bei Nutzung von Standarparametern haben ihre Berechtigung nachgewiesen. Hier ist bspw. die Nucleus Fitting Software (NFS) der Fa. Cochlear Ltd. zu nennen, die bei Nutzung eines Tablet-Computers und eines Wireless- 
PODs sehr flexibel eingesetzt werden kann. Damit ist die Anpassung nicht zwangsläufig an die Laborsituation in einem Audiometrieraum gebunden, sondern kann in sehr unterschiedlichen Umgebungssituationen durchgeführt werden. Es ist nicht zwingend ein AnpassExperte erforderlich, die Anpassung kann im unkomplizierten Routinefall auch im Sinne von Remote Fitting bei angelerntem Personal wie bspw. Hörgeräteakustikern erfolgen. Basis der Anpassung sind automatisch bestimmte CAP-Schwellen sowie punktuell bestimmte Threshold- und Comfort-Level. Botros et al. [77] konnten bei $13 \mathrm{~Pa}$ tienten zeigen, dass sich das Outcome nach Fitting mit der NFS-Software von dem mit der Custom Sound nicht signifikant unterschied.

Zu lösen sind in diesem Zusammenhang Fragen der Qualitätsüberwachung zur Vermeidung von Fehlanpassungen.

Remote Fitting/Remote Care mit Videounterstützung kann die Anpassung entfernt wohnender Patienten unterstützen. Hierbei kommt auch eine Wundkontrolle über Foto oder Videokamera in Betracht ( $\triangleright$ Abb. 6).

Dabei ist der Patient mit dem interagierenden Zentrum über eine Datenfernverbindung verbunden. Der Experte in der implantierenden Klinik oder dem Cl- Zentrum kann den Patienten beobachten und mit ihm interagieren. Ein direkter Zugriff auf das Implantat ist ggf. über eine eingewiesene Fachkraft vor Ort oder ein vom Patienten selbst bedientes Interface möglich. Dadurch lassen sich Feinanpassungen, insbesondere in häuslicher Umgebung, Technikchecks und Upgrades der Software durchführen [78].

Eikelboom et al. [79] haben ein computergestütztes Remote Fitting System für mit MEDEL-Implantaten versorgte Patienten entwickelt und an 11 Patienten evaluiert. Im Durchschnitt dauerte das Remote Mapping 42 Min, während im Vergleich die konventionelle Anpassung $37 \mathrm{Min}$ in Anspruch nahm. In den anschließenden Sprachtests (Ling Six Test, Beantwortung von Fragen) zeigte sich kein signifikanter Unterschied zwischen beiden MAPs. Sechs Patienten präferierten keine der beiden MAPs, 2 bevorzugten die konventionelle MAP und 3 die Remote MAP. Berichtet wurde von zeitlichem Versatz zwischen Audio- und Videokanal, was die Kommunikation des Patienten mit dem Experten erschwerte, weil das Mundbild nicht unterstützend genutzt werden konnte. Hier war die Unterstützung durch die Fachkraft vor Ort erforderlich. Zehn Patienten gaben schließlich an, dass sie zukünftig über Remote Fitting angepasst werden möchte, alle 11 Patienten würden das Verfahren weiterempfehlen.

Kuzovkov et al. [80] berichten sehr positiv über Remote Anpassungen an 33 Patienten in Italien, Schweden und Russland. Involviert waren jeweils ein Anpassexperte auf der Klinikseite, der Patient und ein lokaler Moderator (lokale Fachkraft) auf der Patientenseite. 96,9\% der Patienten waren mit dem Anpassergebnis zufrieden, $100 \%$ sprachen sich für weiteres Remote Fitting aus.

Auch Wasowski et al. [81] bestätigen die Effizienz von Remote Fitting im Vergleich zum Face-to-Face-Fitting. Den Patienten bleiben weite Wege erspart, teilweise kann dadurch die Frequenz der Anpassungen erhöht werden.

Self-Fitting durch den Patienten kann mit automatisierten Algorithmen auf der Grundlage von CAP-Schwellen erfolgen. Der Patient kann Lautstärke, Höhen und Tiefen selbst anpassen, ggf. Comfort- und Threshold Level selbst bestimmen.

In [77] wird das Ergebnis der Selbst-Anpassung mit dem CR110 Remote Assistenten von Cochlear Ltd. mit dem Ergebnis einer Anpassung durch einen audiologischen Experten mit der Custom Sound verglichen und auch hier im Outcome kein signifikanter Unterschied festgestellt.

Ob Fitting beim Experten in der Klinik oder ReHa-Institut, Remote Fitting beim wohnortnahen Sekundärpartner (lokale Fachkraft) oder Self-Fitting - Wireless Programmierung erlaubt heute eine sehr viel komfortablere und für den Patienten bequemere Ankopplung an das Anpasssystem. Insbesondere in der Kinderanpassung ist damit ein großer Schritt in Richtung der Verbesserung der Akzeptanz der Anpasssituation gelungen.

Durch Vernetzung über das Internet bestehen heute Möglichkeiten der Früherkennung von Problemen (bspw. Erhöhung der Elektrodenimpedanzen - ggf. Anzeichen für beginnende Labyrinthitis [78]).

Remote Troubleshooting (z. B. [82]) kann dem Patienten helfen, Probleme mit seinem Soundprozessor oder dem Zubehör selber zu analysieren und zu beheben, ohne einen Servicepartner oder gar die implantierende Klinik aufsuchen zu müssen. Möglicherweise unnö-

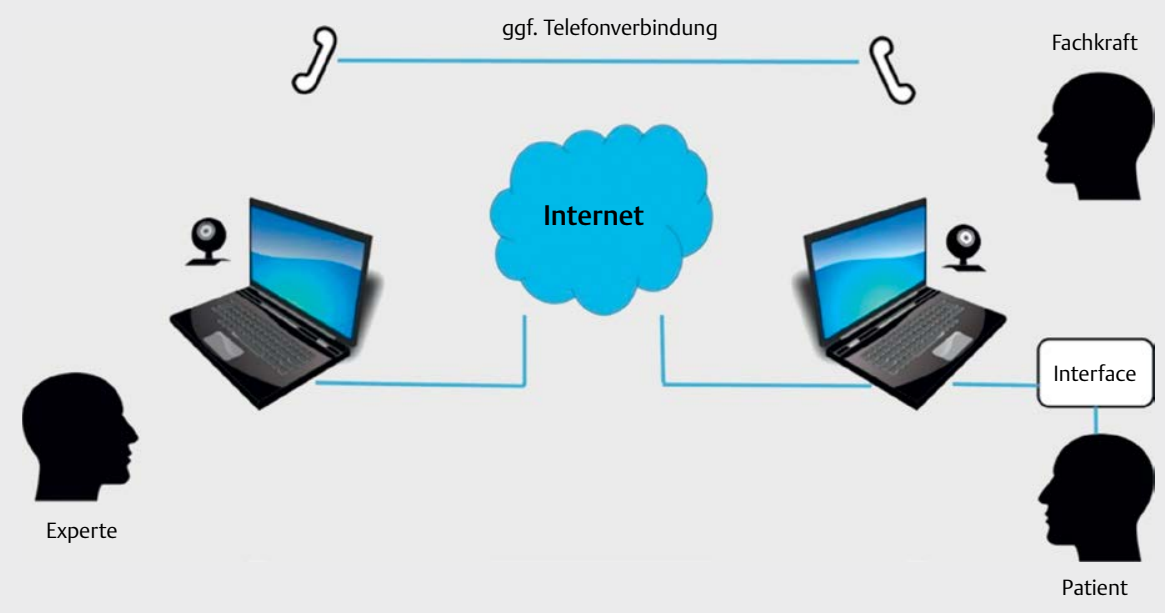

Abb. 6 Remote Fitting. 
tiger Versand von Ersatzteilen oder Austauschprozessoren kann verhindert werden.

Eine sehr entscheidende Fähigkeit moderner Cl-Systeme ist das Data Logging. Es erlaubt im Rahmen der Anpassungen Erkenntnisse über den Gebrauch der einzelnen MAPs, über die Variabilität und zeitliche Wichtung der Hörsituationen des Patienten, über seine Hörgewohnheiten bzgl. der Einstellungen von Lautstärke und Empfindlichkeit und nicht zuletzt über die Nutzungsdauer. Diese Erkenntnisse sind nicht nur für Anpasser und Therapeuten wichtig, sie sind auch für die Cl-Hersteller richtungsweisend.

Erfolgskontollen im Sinne der Erfassung des Sprachverstehens sind ebenfalls telemedizinisch mit entsprechenden Computerprogrammen/Apps möglich. Die Lebensqualität kann über Fragebögen computer- oder App-gestützt erhoben und ausgewertet werden.

„Artifical Intelligence“, „Cloud Connectivity“ und „Wireless Technology“ sind Begriffe, die zeigen, wohin die Reise bei der Cl-Nachsorge geht bzw. heute schon Anwendung finden. Wie bereits in der Einleitung ausgeführt, kann die enge Vernetzung ( $>$ Abb. 5) aller im ReHa-Prozess beteiligten (Patient, implantierende Klinik, ReHa-Institution, Hersteller, Service-Partner, ggf. sekundäre Betreuungseinrichtung) mit effizientem Datenaustausch unter Beachtung der gültigen Normen des Datenschutzes zu Kosteneinsparung, schnellerem und optimalerem Versorgungserfolg, gesteigerter Patientenzufriedenheit, schnellerer Problembehebung, Früherkennung sich anbahnender Probleme und Wissenszuwachs durch BigData führen.

- Abb. 7 zeigt, welchen Stellenwert die einzelnen Bausteine der Cl-Nachsorge in Zukunft haben werden. Insbesondere im Zuge der lebenslangen Nachsorge werden Remote Care und Self Care eine immer größere Bedeutung erlangen. Das entspricht dem Bedürfnis der Patienten nach einer optimalen und sicheren Funktion ihres Cochlea-Implantats ohne Inanspruchnahme langer mit weiten Wegen verbundenen Termine in zentralen Einrichtungen. Dabei besteht trotzdem der Wunsch, dass die Funktion und sich anbahnende Störungen vom Experten überwacht werden, was durch Telemedizin möglich wird. Das entspricht aber auch der Zielsetzung der Kostenträger zur Kostenreduktion und den Zwängen der Kliniken und
ReHa-Einrichtungen zur optimalen Nutzung der Personalressourcen. Der einfach zu versorgende „Standardpatient“ kann damit auf Dauer komfortabler und sogar sicherer betreut werden, während die Experten mehr Zeit für die speziellen Fälle haben, die sehr viel individuelle Zuwendung erfordern.

Kritisch muss diskutiert werden, dass die sehr positiven Aspekte beim Remote- und Self-Fitting nur für Patienten ohne spezielle audiologische und sonstige Besonderheiten gelten. Ein Großteil der alten und sehr alten Patienten hat einen sehr großen individuellen Betreuungsbedarf und ist zum Self-Fitting oder Remote-Fitting in keiner Weise in der Lage. Bei einer nicht unerheblichen Anzahl von Patienten ist nach Erfahrung der Autoren die Nutzung von Auto-CAP nicht möglich, weil bei ihnen zum Erzeugen auswertbarer CAP-Antworten höhere Pulsbreiten erforderlich sind, die von der Auto-Routine nicht angeboten werden. Bei Patienten mit speziellen Problemen wie schlechter Akzeptanz, Verstärkung des Tinnitus unter der Cl-Therapie, Abweichungen von der regulären Tonotopie, Missempfindungen oder Fazialismiterregung bei einzelnen Elektroden usw. sind die automatisierten Verfahren nicht einsetzbar.

Ferner ist eine breitbandige Internetverbindung, wie sie für Remote-Fitting und -Care erforderlich ist, nicht allerorts Standard.

Die Zeitersparnis bei unkomplizierten „Standardpatienten“ durch vereinfachte Fittingverfahren oder Remote Care kann aber erheblich sein und ggf. dem „Problempatienten“ zu Gute kommen.

\section{Digitalisierung in der hörtherapeutischen Folgetherapie}

Auch im Hörtraining spielt Standardisierung und Vergleichbarkeit eine maßgebende Rolle. Diese Forderung wird am besten erfüllt durch die Nutzung computergestützter Hörtrainingsprogramme wie z. B. AudioLog von Flexsoft. Die Überlegenheit gegenüber Life Speech liegt auf der Hand, standardisierte und kalibrierte Sprachund Geräuschausgabe garantieren vergleichbare Übungs- und Testsituationen in verschiedenen Sitzungen und Vergleichbarkeit zwischen verschiedenen Zentren.

Vor allem das isolierte Üben und Testen des implantierten Ohres lässt sich sehr gut und definiert durch direktes Streaming von Hör-

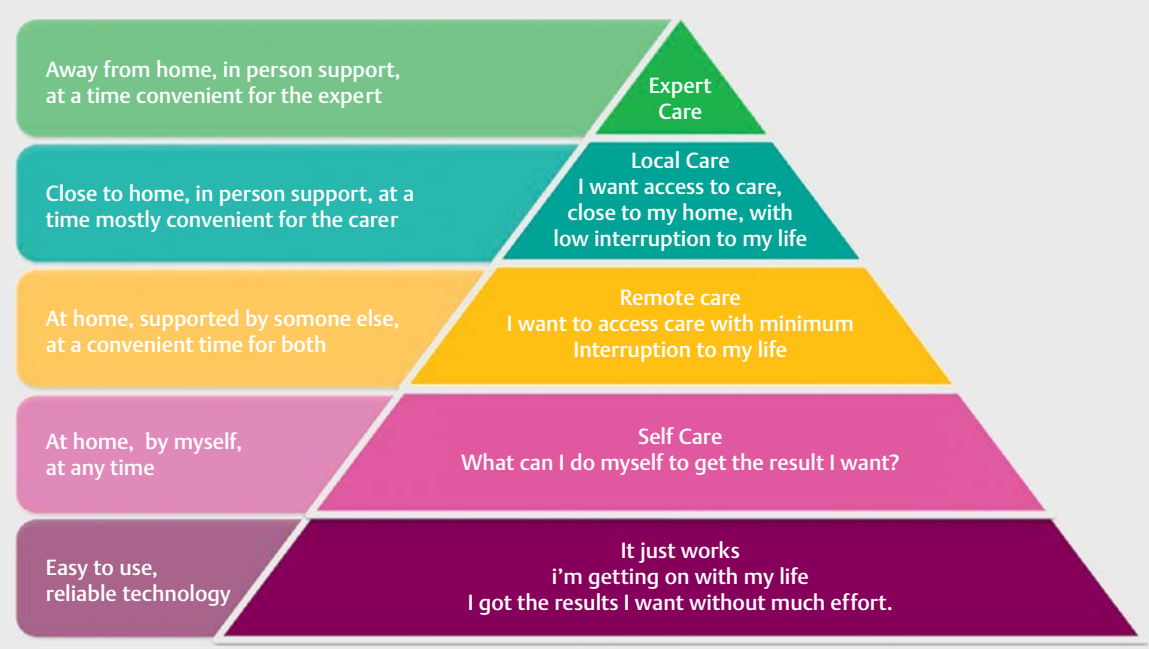

Abb. 7 Maslowsche Bedürfnishierarchie (mit freundlicher Genehmigung von Cochlear Ltd.). 
trainingsinhalten in die Soundprozessoren über Wireless Zubehör erreichen.

Die Ergebnisse des Data Loggings sind sehr hilfreich, um Informationen über die Nutzungsgewohnheiten und das Hörumfeld des Patienten zu erhalten und daraus Tipps zur Programmnutzung und zum Hörverhalten ableiten zu können.

Outcome-Messung bei direkter Einspeisung von Testmaterial in den Soundprozessor (z. B. Auditory Speech Sounds Evaluation (ASSE)) ermöglichen die Testung in Räumen, die keinen anspruchsvollen akustischen Voraussetzungen genügen und garantieren die isolierte Testung des versorgten Ohres ohne Mit- oder Überhören auf dem Gegenohr.

Cl-Nutzer können an telemedizinischen Rehabilitationsprogrammen über Telefon oder Internet teilnehmen. Die Remote-Reha-Sitzungen ähneln den Sitzungen von Face-to-Face und ermöglichen es Patienten, sich ohne umfangreiche Reisebelastung mit Rehabilitationsexperten auszutauschen.

\section{Vorteile der Digitalisierung für den Patienten in der täglichen Cl-Nutzung}

Die Digitalisierung hat den Umgang der Patienten mit dem CochleaImplantat gravierend verändert. Während die Möglichkeit der Lautstärkeverstellung und der Auswahl zwischen mehreren Programmen bereits bei den Taschen-Sprachprozessoren möglich war, änderten sich die Bedienmöglichkeiten bereits mit den ersten Fernbedienungen deutlich.

Heute werden von Gerätegeneration zu Gerätegeneration die individuellen Eingriffsmöglichkeiten für den Patienten immer umfassender und der Umgang mit dem $\mathrm{Cl}$ zunehmend komfortabler.

Signalvorverarbeitung, teilweise mit automatischer Situationserkennung und entsprechender automatischer Anpassung mit dem Ziel der bestmöglichen Beherrschung komplexer Hörsituationen (Bsp. Scan-Programm, Fa. Cochlear Ltd.), wird von den meisten Patienten erfolgreich verwendet.

Manuelle Möglichkeiten der Anpassung an die jeweilige Hörsituation über Fernbedienungen sind seit geraumer Zeit selbstverständlich.

Heute hat die übergroße Mehrheit der Patienten ein Smartphone und nutzt dieses sehr aktiv. Somit ist die Einbindung des Smartphones in die Sprachrozessorbedienung über SmartApps nur zwangsläufig. Dabei gehen die Möglichkeiten über die bekannten Einstellmöglichkeiten mit den bisherigen Fernbedienungen weit hinaus:

- Programmwahl

- Einstellung von Lautstärke und Mikrofonempfindlichkeit

- MVBT (Cochlear Ltd.): Einstellbarkeit von MasterVolume, Bass, Treble

- Audiostreaming

- Bereitstellung von Nutzungsdaten für den Patienten (Anteil der Tragezeit mit Sprache, Anzahl von „Coil-Off's“)

- Kontrolle des Batterieladezustands

- Soundprozessorsuche

Wireless-Zubehör hat die Nutzung der Cochlea-Implantate revolutioniert. Ob es der störungsfreie Musikgenuss, das gute Hören beim Fernsehen unabhängig vom Sitzplatz ist oder die Möglichkeit das vorausfahrende $\mathrm{Cl}$-Kind auf dem Fahrrad akustisch erreichen zu kön- nen oder die Möglichkeit den Referenten in einer Vortragsveranstaltung trotz größeren Abstands gut zu verstehen, die Möglichkeit des Audiostreamings verbessert die Hörsituation der Patienten gravierend. Das von der Handhabung her komfortable und störungsfreie Telefonieren ohne das Telefon überhaupt aus der Tasche nehmen zu müssen bei Nutzung eines Telefonadapters oder direkt über bluetooth ist für die Patienten ein riesiger Gewinn. Sowohl beim Hörtraining in der Nachsorgeeinrichtung als auch beim abendlichen Üben mit dem Hörbuch ist die Möglichkeit, das Soundsignal direkt in den Sprachprozessor zu streamen, nicht mehr wegzudenken.

Bereits im Abschnitt über das Hörscreening wurde auf die fast unüberschaubare Anzahl der verfügbaren Health-Apps für Android und iOS, darunter Hörtest-Apps, Otoscope-Apps, Hörtrainings-Apps u. a. hingewiesen.

Auch im Bereich der Basis- und Folgetherapie können zur orientierenden Erfolgskontrolle durchaus Apps zur Anwendung kommen, aber auch hier gilt die von Bright et al. [83] formulierte Erkenntnis, dass die derzeit verfügbaren Hörtest-Apps kein Ersatz für den Goldstandard, die durch einen Spezialisten durchgeführte PTA, sein können. Aufgrund der niedrigen Kosten, der Portabilität und leichten Zugänglichkeit können derartige Apps aber durchaus therapiebegleitend orientierend sinnvoll sein, so auch in Ländern mit unzureichender medizinischer Breitenversorgung.

Hier spielt die Hardware natürlich eine entscheidende Rolle. Es bedarf hierzu kalibrierter Schallwandler mit entsprechend linearem oder zumindest bekanntem und in der App ausgleichbarem Frequenzgang.

Des Weiteren werden Apps angeboten, die das individuelle Hörtraining mit dem Smartphone erlauben, die Ergebnisse werden protokolliert, der Trainingserfolg anhand von Statistiken ausgewiesen. Die im deutschsprachigen Raum derzeit bekanntesten Apps sind Asklepios Hörtraining vom Hanseatischen Cochlea-Implant-Zentrum und Listen UP! der Fa. MED-EL. Diese Apps ersetzen nicht das fachlich begleitete Hörtraining in einer Nachsorgeeinrichtung, können aber bei kontinuierlicher Anwendung den Rehabililationsprozess durchaus unterstützen und den Patienten motivieren.

Wie bereits in der Einleitung dargelegt, sind Datenspeicherung in zentralen Datenspeichern sowie Vernetzung bei der Betreuung der Cl-Patienten heute durchaus schon Realität, so die Vernetzung von implantierender Klinik, Nachsorgeeinrichtung, Service-Partner (z. B. Hörgeräteakustiker) und Cl-Hersteller. Damit sind bspw. Serviceanfragen sehr effizient zu lösen ( $\triangleright$ Abb. 8). Der Serviceerbringer muss nicht erst recherchieren, bei wem die aktuelle MAP vorliegt, den Betreffenden erreichen und warten, bis ihm die aktuellen Daten zugeschickt werden, wodurch sich die Lieferung des Ersatzteils leicht 1-2 Werktage verzögern könnte. Bei jeglicher Art der Vernetzung der beteiligten Partner, bei der patientenbezogene Daten ausgetauscht werden, sind natürlich die geltenden Vorschriften des Datenschutzes subtil zu beachten.

Verbesserung des bilateralen Hörens durch digitale Signalverarbeitung und Steuerung

Ziel jeglicher Versorgung mit Hörhilfen ist ein möglichst gutes binaurales Hören. Speziell bei der Versorgung von Patienten mit 2 CochleaImplantaten oder bimodaler Hörlösung ( $\mathrm{Cl}$ auf der einen, $\mathrm{HG}$ auf der anderen Seite) steckt in der modernen Digitaltechnik ein weitgehen- 


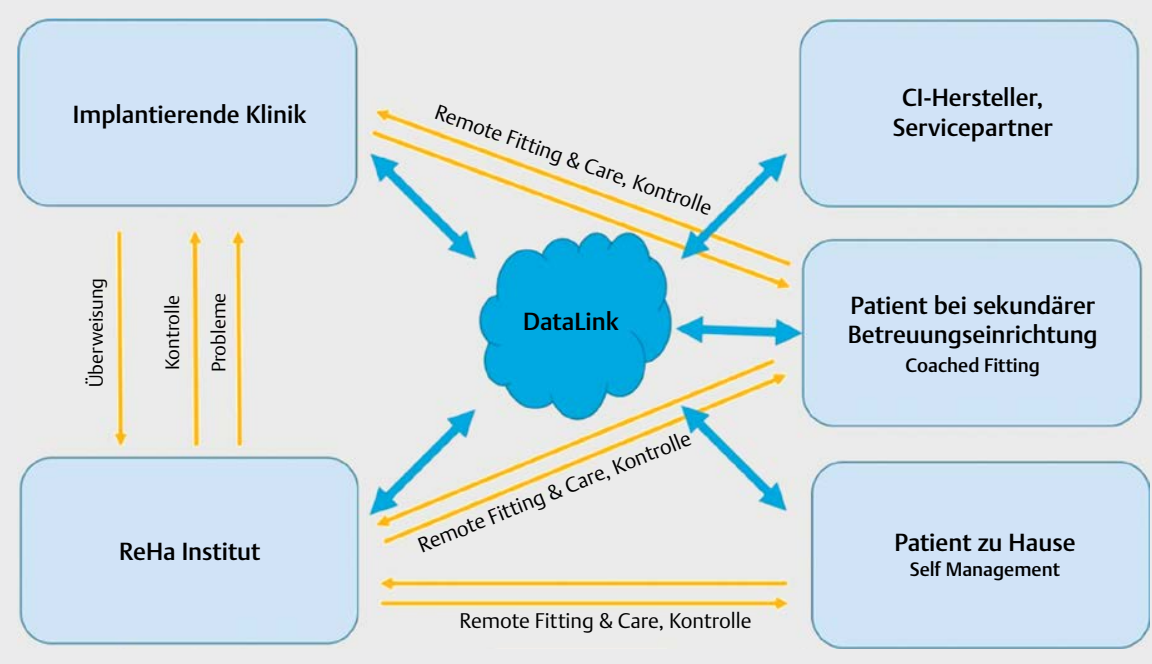

Abb. 8 Vernetzung im Nachsorgeprozess.

des Optimierungspotenzial. So ist es bereits möglich, dass 2 Sprachprozessoren oder ein Hörgerät und ein Sprachprozessor miteinander kommunizieren und zusammenarbeiten. Dadurch kann die Fokussierung auf einen Sprecher im Störgeräusch wesentlich optimiert werden (StereoZoom), kann die Richtung, ,aus der gehört werden soll“ komfortabel festgelegt werden (ZoomControl), kann der Ton des Telefons problemlos auf beiden Seiten gehört werden, obwohl man sich das Telefon an nur ein Ohr hält (DuoPhone), können beide Seiten mit nur einem Tastendruck verstellt werden (QuickSync) [84].

Bei beidseitiger Taubheit, aber nur einseitiger Versorgbarkeit mit einem $\mathrm{Cl}$ besteht die Möglichkeit einer sehr komfortablen CROS-Versorgung über Naida Link CROS [85].

\section{Nachsorge - Digitaliserung - Cl \\ Was bedeutet "Cl-Nachsorge“?}

Die Cl-Rehabilitation gliedert sich in mehrere Bestandteile (siehe Absatz „Digitalisierung in der postoperativen Phase“), wobei die lebenslange Nachsorge einen integralen Bestandteil einnimmt. Die Nachsorge beginnt nach Empfehlungen der DGHNO beim Erwachsenen ca. 1 Jahr postoperativ und bei Kindern ca. 4 Jahre postoperativ. Sie knüpft nahtlos an die Folgetherapie an und dauert lebenslang an.

Die Cl-Nachsorge strukturiert sich in 3 Abschnitte, die audiologische, die technische und die medizinische Nachsorge resp. Kontrolle. Das Ziel der Nachsorge soll die Stabilisierung und Optimierung der Kommunikationsfähigkeit sein [13].

\section{Herausforderung aus Patientensicht}

Für die lebenslange Nachsorge von mit Cochlea-Implantaten versorgten Patienten sind hohe logistische und personelle Aufwendungen notwendig. Der Patient wird möglicherweise mit hohem zeitlichen Aufwand und damit verbundenem Arbeitsausfall, kostenintensivem Transfer sowie mit Störungen im Familienleben belastet [86]. Zu berücksichtigen sind auch Lifestyle-Aspekte, d. h. der Patient wünscht aus persönlichen Gründen eine ortsungebundene Nachsorge.

\section{Herausforderung aus Kliniksicht}

Durch die steigende Anzahl Hörgeschädigter (siehe Einleitung) und der resultierenden steigenden Anzahl Cl-Versorgter besteht vor dem Hintergrund eines zunehmenden Kostendrucks die Notwendigkeit der Prozessoptimierung.

Zielführende Strategien für den Erhalt einer maximalen Versorgung sind erforderlich. Der Anspruch dabei ist, die Höchstlevelmedizin allen Patienten zugänglich zu machen und Kompetenzen in Behandlungszentren weiter auszubauen.

Die Digitalisierung kann dabei einen wesentlichen Beitrag leisten, jedoch ist bei der Einführung internetbasierter Angebote der Ausbau der Internet-Bandbreite zu berücksichtigen. Ballungszentren stellen hierbei keine Probleme dar. Entlegenere Regionen wurden hingegen in den vergangenen Jahren wiederholt vom Breitbandausbau benachteiligt [87]. So ist das beschlossene Ziel der derzeitigen Regierungskoalition aus aktuell 33,2 Mio. Breitbandanschlüssen landesweit bis 2025 ein flächendeckendes Angebot aufzubauen [88].

\section{Lösungsansätze in der Cl-Nachsorge}

Saunders und Chisolm [89] definierten 4 Anwendungs- und Entwicklungsbereiche der „Tele-Audiologie“ (vgl. Einleitung), die im Rahmen der Nachsorge konzeptuell Anwendung finden, um diesen Anforderungen durch Einsatz digitaler Medien gerecht zu werden. Diese Tele-audiologischen Möglichkeiten sind überlappend in den 3 Nachsorgemodalitäten technische, medizinische und audiologische $\mathrm{Cl}$ Nachsorge einsetzbar.

Im Rahmen der technischen Nachsorge können per Video- oder Telefonkonferenz bspw. Fernberatungen oder CAP-Messungen online vorgenommen werden. Ebenso wäre die Beratung als „store and forward“ (vgl. Anwendungsbereiche Tele-Audiologie) und die CAPMessung im Rahmen eines „remote monitoring“ denkbar.

Die medizinische Nachsorge als rein telemedizinisches Angebot unterliegt spezifischen Regularien, die an anderer Stelle dieses Referatebandes detailliert aufgeführt werden. Bei isolierter Betrachtung der technischen Möglichkeiten bietet sich die Videokonferenz mit „face-to-face“ Kontakt an. Klinische Untersuchungen könnten auch 
bei der Nachsorge, wie bereits im Kapitel Screening beschrieben, über sog. Otoskopie-Apps abgebildet werden [90].

Die wahrscheinlich interessantesten Möglichkeiten bei Betrachtung der Digitalisierung ergeben sich für die audiologische Nachsorge, die regelhaft bei Auffälligkeiten in der technischen Nachsorge stattfindet. Neben den bereits erwähnten Möglichkeiten des persönlichen Patientenkontaktes sind bspw. „mobile health“ Angebote zur Selbstkontrolle und zum Selbstmanagement von Sprachprozessoreinstellungen sowie für Hör- und Sprachtest-Kontrollen hilfreich.

\section{Hörtraining in der $\mathrm{Cl}-$ Nachsorge}

Das Hörtraining erfolgt in wesentlichen Bestandteilen im Rahmen der Basis- und Folgetherapie nach Cl (vgl. Kapitel Basistherapie). Dennoch bedeutet die Versorgung mit einem $\mathrm{Cl}$ lebenslanges Hörtraining und somit sind die Entwicklung des Hörtrainings im Rahmen der Nachsorge nicht uninteressant.

Zur Verbesserung des Sprachverständnisses werden von den führenden $\mathrm{Cl}$-Herstellern und aus anderen Quellen Applikationen angeboten [91]. Diese erwachsen aus der Evidenz der auditorischen Rehabilitation von Eins-zu-Eins-Situation und bieten die Möglichkeit eines auditorischen oder audiovisuellen Trainings. Computerbasierte Anwendungen steigern nachweislich die Motivation zum Hörtraining, was wahrscheinlich der größte Nutzen dieser Anwendungen auf dem Weg zur Verbesserung der Sprachwahrnehmung ist $[5,89]$.

\section{Remote-Care-Netzwerke}

Über sog. Remote-Care-Netzwerke kann die Expertise des Behandlungszentrums von verbundenen, entfernten Einrichtungen, z. B. weniger spezialisierten Kliniken, Akustikern oder Rehazentren abgerufen werden und auch interdisziplinär stattfinden [92]. Diese angeschlossenen Behandlungseinrichtungen sollten einen primären Service wie bspw. Kontrollen des Hörvermögens oder technische Checkups selbst abdecken und bei Unklarheiten und Problemen die Expertise der übergeordneten Klinik abrufen können. Technisch kann dies über einen webbasierten „face-to-face“ Kontakt erreicht werden. Der zugeschaltete Experte kann Einstellungen am Sprachprozessor vornehmen und Daten, wie bspw. Nutzungsdaten, abrufen.

Folgende Technologien werden ebenfalls in Remote-Care-Netzwerken genutzt:

- Kabelfreie Technologien, z. B. zum Echtzeitdatenabgleich mit dem Smartphone

- Künstliche Intelligenz, bspw. zur automatisierten Einstellung von Cochlea-Implantaten sowie Kontrollen von Einstellungen in der Nachsorge

- Cloud-basierte Netzwerke zum Datenaustausch mit bspw. spezialisierter Klinik - Patient - Rehaeinrichtung - nachgeordneter Klinik

Die Leistungen von Remote-Care-Netzwerken müssen standortspezifisch angepasst werden. So ist in ländlich geprägten Regionen der Wissenstransfer in die Fläche erforderlich und führend, um bspw. unnötige Fahrwege für Patienten zu minimieren und damit verbundene zeitliche und monetäre Aufwendungen zu reduzieren. In Ballungszentren ist dies freilich ebenfalls erforderlich, jedoch ist hier aufgrund der hohen Dichte an spezialisierten Gesundheitseinrichtungen der Fokus bei eher immobilen Patienten oder zum Datenaustausch mit standortfernen Reheinrichtungen zu sehen.

\section{Evidenz der telemedizinischen $\mathrm{Cl}$-Nachsorge}

Aus der Universität von Southampton und der Arbeitsgruppe von Cullington [86, 93] wurde 2018 eine 2-armige kontrollierte, randomisierte Studie (RCT) mit dem Ziel der Evaluation der Langzeitnachsorge über eine internetbasierte Fernnachsorge vorgestellt. Beide Studienarme enthielten je 30 Patienten und liefen über einen Zeitraum von 6 Monaten. Getestet wurden das Sprachverstehen, das Patienten-Selbstmanagement mit dem $\mathrm{Cl}$, die selbstberichtet subjektive Gehörwahrnehmung und die Lebensqualität. Die Autoren berichten, dass die remote-care Gruppe 6 Monate nach Studienbeginn ein signifikant besseres Sprachverstehen und einen höheren Wert beim Patienten-Selbstmanagement aufwiesen, d.h. die Patienten hatten einen höheren Wissenstand über das $\mathrm{Cl}$ und berichteten insgesamt bessere Fähigkeiten beim Umgang mit dem $\mathrm{Cl}$. Dahingegen berichtete die Kontrollgruppe signifikant schlechtere Werte bei der subjektiven Selbstbeurteilung des Hörvermögens. Die Lebensqualität sei nach 6 Monaten in beiden Gruppen gleich gewesen. Die Limitationen dieser nach dem Wissen der Autoren ersten RCT zur Nachsorge von Cl-Patienten wurde von den Autoren resümiert, dass die telemedizinische Fernnachsorge keine Universalmöglichkeit für alle Cochlea-Implantat-Patienten darstelle, sondern vielmehr im Rahmen des shared decision-making mit dem Patienten individuell abgestimmt werden solle.

\section{Outcome}

Die Machbarkeit der internetbasierten Nachsorge wurde gezeigt, die gleichzeitig zur Erhebung des Outcomes genutzt werden kann. Fragebogen-gestützte Auswertungen wie bspw. die Lebensqualität oder das subjektive Hörervermögen, können unproblematisch online angeboten werden. Selbst bei einem Termin in der Klinik können die Daten elektronisch bspw. über ein Tablet abgegriffen und über eine große Datenbank zugänglich gemacht werden. Der Prozess des Big Data erfordert jedoch vorherige Absprachen zur Standardisierung, die im Rahmen einer Konsensbildung möglichst weitreichend erwirkt werden sollten. Die Probleme von zu kleinen Studiengruppen wurden in der Vergangenheit oft angeführt. Die ausgeprägte Heterogenität der Erhebungsinstrumente zwischen nationalen und internationalen Zentren ist dafür ausschlaggebend. In eigenen $\mathrm{Ar}$ beiten schlugen wir wiederholt die psychometrische Testbatterie der Charité - Berlin vor [5-8, 10-12, 20], deren Lebensqualitätsinstrument der NClQ in das im April 2018 erschienene Weißbuch „Cl-Versorgung" aufgenommen wurde.

Insbesondere für die Bestimmung kleiner Effekte sind große Fallzahlen notwendig. Prädiktoren für die zukünftige Cl-Versorgung könnten bestimmt und Indikationen bestätigt werden. Im Rahmen des „data logging“ bestünde die Möglichkeit Outcome-Daten mit anderen patientenbezogenen Daten zu verknüpfen und somit durch ausführliche Analysen individuelle Konzepte für den Patienten, letztendlich auch durch Weiterentwicklung der künstlichen Intelligenz zu erreichen.

\section{Perspektiven}

Telemedizinische Behandlungs- und Nachsorgekonzepte sind aus anderen Bereichen der Medizin, bspw. Kardiologie oder Psychiatrie länger bekannt und weiter fortgeschritten. In der Cochlea-Implantat-Versorgung steht die Entwicklung noch am Anfang einer aus Sicht der Autoren vielversprechenden Neuerung der existierenden Nach- 
sorgemodalitäten. Basierend auf den Aussagen von Cullington und Mitarbeitern ist zu erwarten, dass die Telemedizin im Cl-Bereich ein zusätzliches Tool sein wird, es jedoch den vor-Ort-Termin, zumindest derzeit, nicht komplett ersetzen kann [86,93]

\section{Innovationscluster Interaktive Mikro- implantate (INTAKT)}

Verbundkoordination: Fraunhofer-Institut für Biomedizinische Technik IBMT

Tinnitussuppression

Am Ende dieses Referates möchten wir ein Forschungsprojekt unserer Klinik im Rahmen des INTAKT Verbundprojektes vorstellen, dass sich inhaltlich perfekt in die Thematik Hörrehabilitation im Zeitalter der Digitalisierung einreiht und illustriert, wie die Zukunft im Bereich der Hörimplantate aussehen könnte.

Das Ziel des Projektes INTAKT ist die Entwicklung, Fertigung, Charakterisierung und präklinische Evaluierung einer neuen Generation von aktiven, vernetzten Implantaten. Diese verfügen über Schnittstellen, die Ärzten und Patienten einen leichten Informationszugriff zur partizipativen Entscheidungsfindung erlauben. Voraussetzung dafür ist die bedarfsgerechte, transparente Darstellung aller erforderlichen Informationen über Zustand und Funktionalität der Implantate einschließlich ihrer Schnittstellen zum biologischen Gewebe. Diese neuen Interaktionsmöglichkeiten gestatten durch Anpassung der Parameter und Modi an die jeweiligen Bedürfnisse des Patienten eine Optimierung und Funktionserweiterung der implantierten Systeme und bieten damit die Möglichkeit der personalisierten individuellen Patientenversorgung durch Implantate.

Zur Realisierung dieses innovativen Ansatzes des Projektes INTAKT wird ein Netzwerk aus bis zu 12 interaktiven Mikroimplantaten entwickelt, aufgebaut und präklinisch getestet. Die intelligente Kommunikation sowohl zwischen den Implantaten als auch mit der zentralen externen Kommunikationseinheit und die Bewertung der erfassten Signale erfolgt auf der Grundlage von Prinzipien der Informationsverarbeitung, d. h. Filterung von Signalen, komprimierende Datenverarbeitung, Mustererkennung, Standard-Routinen usw. Dies ermöglicht eine zeitliche wesentlich bessere Synchronisation einer Vielzahl von Aktivitäten. Damit kommen die Mikroimplantate in ihrer Funktionalität physiologischen Gegebenheiten weitestgehend nahe und können so Beeinträchtigungen der Betroffenen aufgrund von funktionalen Defiziten besser und komplexer kompensieren.

Die Intelligenz des Systems entsteht aus der Kopplung interner und externer Systeme auf der Basis eines sicheren Datenaustausches. Ein weiterer Vorteil gegenüber herkömmlichen Implantaten besteht darin, dass das Netzwerk von Mikroimplantaten nicht nur an einer einzelnen Stelle aktiv ist, sondern die Funktionalität größerer zusammenhängender Gewebeabschnitte und Organe in ihrer Gesamtheit berücksichtigt. Dadurch können pathologische Veränderungen nicht nur punktuell, sondern in physiologischer Weise interaktiv an mehreren Stellen therapeutisch beeinflusst werden.

Die vernetzten Implantate kommunizieren über äußere Schnittstellen bei Bedarf mit dem Patienten und dem medizinischen Fachpersonal. Dadurch wird eine personalisierte Anpassung des Implantatnetzwerkes an die momentanen Bedürfnisse des Patienten möglich. An Stelle rigider Stimulationalgorithmen tritt also eine interaktive Bedarfssteuerung. Solche individualisierten, multilokulären und interaktiven
Implantate bilden die Grundlage für eine Vielzahl von relevanten Anwendungsszenarien.

Eine besondere Rolle innerhalb dieser Thematik kommt der Tinnitussuppression mittels Mikroimplantaten zu. Hier eröffnen sich durch neue technische Möglichkeiten Behandlungsoptionen, welche eine Umsetzung in eine Anwendung zur Behandlung von Patienten möglich macht. Weitere Anwendungsbereiche betreffen die Therapie von Funktionsstörungen des Verdauungstraktes und die Entwicklung eines neuromuskulären Stimulators zur Realisierung von Greiffunktionen.

\section{Problembeschreibung}

Tinnitus ist definiert als die subjektive Wahrnehmung eines Geräuschs bei Fehlen einer äußeren Schallquelle. Es handelt sich nicht um ein einheitliches Krankheitsbild, sondern um ein Symptom, das durch vielfältige Ursachen hervorgerufen werden kann (z. B. Hörstörungen, kardiovaskuläre oder neurologische Erkrankungen, Diabetes oder Tumoren).

Tinnitus ist häufig mit Hörstörungen assoziiert, kann aber auch als unabhängiges Symptom auftreten. Als ursächlich werden Dysfunktionen im Hörsystem angenommen. Die aktuelle Leitlinie „chronischer Tinnitus“ fasst den derzeitigen Wissensstand zur Pathophysiologie des Tinnitus zusammen [94] wobei nach den neuesten psycho- und neurophysiologischen Untersuchungen zu den Mechanismen neuronaler Plastizität sowohl periphere als auch zentrale Veränderungen eine Rolle spielen.

Die Behandlungsbedürftigkeit wird durch das Auftreten eines deutlichen Leidensdrucks und durch vorhandene oder entstehende Komorbiditäten bestimmt. Der Leidensdruck kann individuell sehr unterschiedlich ausgeprägt sein und korreliert nicht mit der Tinnitusfrequenz oder Lautstärke [95]. Die großen individuellen Unterschiede sind v. a. durch die unterschiedliche Ausprägung von Begleitsymptomen und Erkrankungen wie z. B. Depressionen, Schlaf- und Konzentrationsstörungen erklärbar [96].

Der entscheidende Faktor für eine therapeutische Intervention im chronischen Stadium ist der Schweregrad des Tinnitus. Für den chronischen Tinnitus gibt es keine kausale Heilungsmethode. Es werden v. a. kognitive und multimodale Verhaltenstherapien angewendet, damit es zu keinen schwerwiegenderen Komplikationen durch die Tinnitusbelastung kommt. Darüber hinaus können hörtherapeutische Interventionen wie Trainings, Hörgeräte oder Cochlea-Implantate zum Einsatz kommen [94-97].

Dennoch kann es bei Patienten mit einer hohen Tinnitusbelastung auch bei Anwendung aller therapeutischen Optionen zu einer gravierenden Einschränkung der Arbeitsfähigkeit und zu einer psychischen Dekompensation kommen. Dies ist neben einer enormen Einschränkung der Lebensqualität für den Patienten auch mit hohen sozioökonomischen Kosten verbunden. Ein verlässlich wirksames Verfahren zur Behandlung des Tinnitus wäre damit von erheblicher medizinischer als auch sozioökonomischer Bedeutung.

\section{Thematische Zielsetzung}

Epidemiologische Untersuchungen haben gezeigt, dass die Tinnitusprävalenz bei 5-15\% liegt [98]. In Deutschland leiden ca. 10 Mio. Menschen unter einem Tinnitus. Bei 10\% dieser Betroffenen besteht ein Behandlungsbedarf. In den USA beträgt allein in der Gruppe der 
US- Veteranen der Umfang der anfallenden Kosten für die Behandlung von Tinnitus $1 \mathrm{Mrd}$. US- Dollar.

Schon seit den 70er Jahren werden Therapieansätze verfolgt, bei denen eine Beeinflussung des Tinnitus durch eine Elektrostimulation der Cochlea erzielt werden kann.

Die Auswirkungen einer elektrischen Stimulation im Rahmen der $\mathrm{Cl}$-Versorgung auf den Tinnitus sind dagegen in zahlreichen Studien untersucht worden. Die aktuelle Literatur belegt die positiven Effekte des $\mathrm{Cl}$ auf den Tinnitus bei einer großen Anzahl von Cl-Nutzern $[6,8,11,12$, 99-105]. Eigene Studien zeigen, dass das $\mathrm{Cl}$ über die Verbesserung des Sprachverstehens hinaus, auch zu einer Verbesserung der Lebensqualität, der Tinnitus,- und Stressbelastung und psychischer Komorbiditäten führt [5-9, 11, 12, 41, 105].

Davon abgeleitet ist die thematische Zielstellung der Entwicklung eines speziellen extracochleären Implantats für Tinnitus Patientenunabhängig von der Hörsituation, überaus relevant.

Die technischen Möglichkeiten der Miniaturisierung ermöglichen heute neue Wege der Übertragung der Elektrostimulation. Physiologisch soll der Effekt auf den Tinnitus durch eine Synchronisierung der afferenten Signale der Cochlea sowie die Unterstützung der zentralen Neuromodulation durch Modifikation der afferenten Signale erzielt werden. Die individuell angepasste Stimulation führt zu einer bedarfsgerechten individualisierten Suppression des Tinnitus. Bei beidseitiger audiologischer Symptomatik ist eine Kommunikation der Stimulationseinheiten zur Anpassung der Stimulationsparameter erforderlich. ( $\triangleright$ Abb. 9)

\section{Ziele, Rolle und Aufgaben im Projekt}

An diesem 13,5 Mio. Euro Projekt sind folgende universitäre und außeruniversitäre Forschungsinstitute, kleine, mittelständige Unternehmen und Großunternehmen beteiligt: Fraunhofer Gesellschaft (IBMT), Universitätsmedizin Mainz, Universitätsklinikum Heidelberg, Charité-Universitätsmedizin Berlin, Universität Mannheim, Technische Universität Ilmenau, GeSiM Gesellschaft für Silizium-Mikrosysteme $\mathrm{mbH}$, inomed Medizintechnik $\mathrm{GmbH}$, Soventec $\mathrm{GmbH}$, Wild- design GmbH \& Co. KG, IL Metronic Sensortechnik GmbH, Glück Engineering $\mathrm{GmbH}$, Würth Elektronik $\mathrm{GmbH} \&$ Co. KG, VARTA Microbattery $\mathrm{GmbH}$, Heraeus Medical Components, CeramTec-ETEC $\mathrm{GmbH}$ and CETECOM ICT Services GmbH. Der Klinik für Hals-NasenOhren-Heilkunde, Charité-Universitätsmedizin Berlin fallen folgende Aufgabenfelder zu:

- Ziel des Teilprojektes ist die Erfassung von Stimulationsparametern zur elektrischen Suppression des Tinnitus sowie zur Charakterisierung der Patientenkohorte

- Evaluierung eines durch die Projektpartner erstellten Implantates hinsichtlich der Implantationsmöglichkeit und Überprüfung des proof of concept

- Testung der in den klinischen Untersuchungen gewonnen Stimulationsparameter im Tiermodell

- Die Rolle der HNO- Klinik der Charité besteht in der klinischen Erfassung der Stimulationsparameter zur Tinnitus-Suppression und in der Durchführung der Tierexperimente.

Die Aufgabe umfasst die Durchführung und Bewertung der klinischen Studie, die Durchführung und Bewertung der tierexperimentellen Versuche und die Anpassung sowie ggf. den Abbruch der Studie bzw. von Teilstudien in Abhängigkeit von den im Rahmen des Projektes gewonnenen Ergebnissen.

Die Neuheit und Attraktivität des Lösungsansatzes liegt in der Kombination einer klinischen Studie mit Evaluierung und Präselektion einer klinisch heterogenen Patientenkohorte (Tinnituspatienten) und der Anwendung dieser Daten im Tiermodell unter Verwendung eines innovativen Implantatsystems.

Das Besondere an dem Verfahren ist die Testung eines zu entwickelnden Implantprototypen, der auf klinisch einfach durchzuführende Weise zur Behandlung eines bis dato schwer behandelbaren Erkrankungsbildes führen kann (unmet need).

Obgleich die Idee einer Elektrostimulation zur Behandlung des Tinnitus schon vor längerer Zeit beschrieben wurde, ist die klinische Umsetzung bislang an der Entwicklung klinisch anwendbarer Syste-

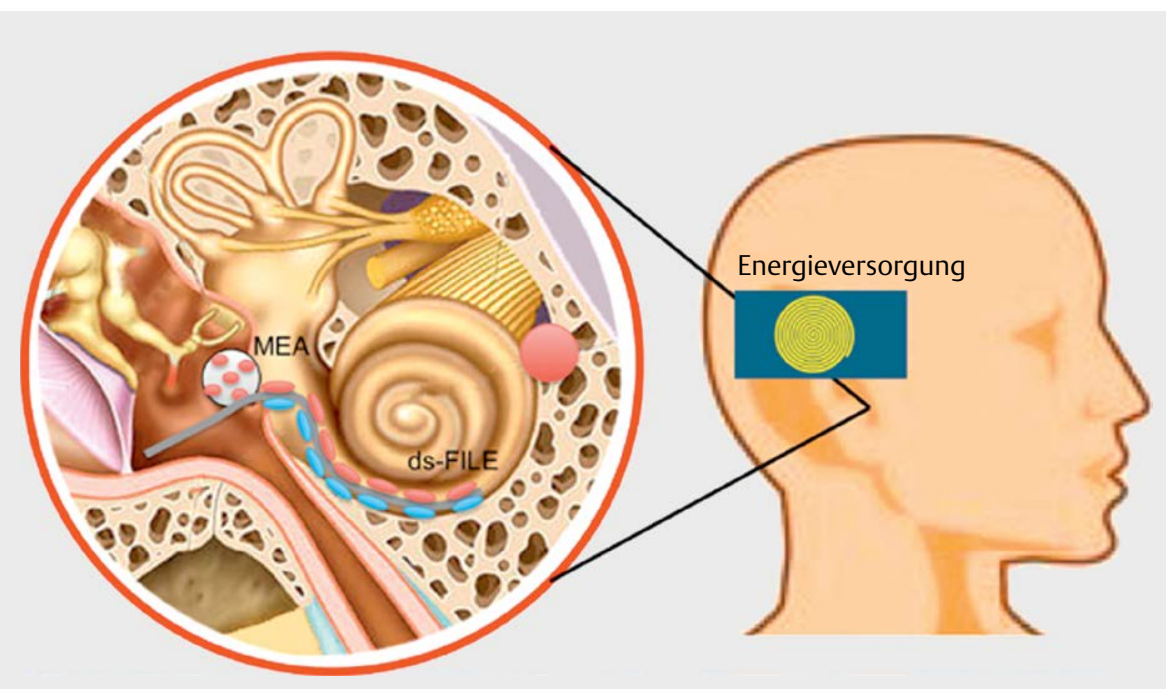

Abb. 9 Untersuchungen zur Suppression des Tinnitus durch elektrische Stimulation unter Einsatz eines Multielektrodenarrays (MEA) am runden Fenster oder einer Mikro-elektrode (ds-FILE, double sided - Filament Electrode). Quelle: „Forschungsprogramm zur Mensch-Technik-Interaktion: Technik zum Menschen bringen“ des BMBF, Verbundprojekt INTAKT, Förderkennzeichen 16SV7875. 
me zur Elektrostimulation gescheitert: Hier könnten die im Zuge des Innovationsclusters INTAKT zu erstellenden Implantate neue Möglichkeiten eröffnen.

\section{Schlussbemerkungen}

In diesem Referat werden die aktuellen und zukünftigen Entwicklungen digitaler Anwendungen aus dem Blickwinkel einer Cochlea-Implantat $(\mathrm{Cl})$ versorgenden Klinik dargestellt und besprochen.

Durch die steigende Anzahl von Menschen mit Hörstörungen, u. a. bedingt durch die demografische Entwicklung, die erweiterten Cl-Indikationen verbunden mit einer hohen Variabilität v. a. bei binauraler Versorgung mit Hörsystemen und der daraus resultierenden steigenden Anzahl Cl-Versorgter besteht vor dem Hintergrund begrenzter Ressourcen die Notwendigkeit neue Wege zu beschreiten.

Auch für die lebenslange Nachsorge von mit Cochlea-Implantaten versorgten Patienten sind hohe logistische und personelle Aufwendungen notwendig. Zu berücksichtigen sind auch Lifestyle-Aspekte, $d$. h. viele Patienten wünschen sich eine ortsungebundene Nachsorge, die sich gut in ihren Alltag integrieren lässt. Die Nutzung digitaler Medien eröffnet auch für die in der Mobilität eingeschränkten Menschen mit Hörstörungen oder weitem Anfahrtsweg bis zur nächsten spezialisierten Klinik die Möglichkeit mit Cochlea-Implantaten versorgt zu werden und die Barriere des vor-Ort-Kontaktes zu überwinden.

Ziel der Bemühungen aller am Rehabilitationsprozess Beteiligter ist die Erhöhung der Qualität der Cl-Versorgung bei gleichzeitiger Erhöhung der Effizienz der eingesetzten Mittel. Hier kommt der Digitalisierung all dieser Prozesse eine Schlüsselrolle zu.

Das Referat illustriert vielfältige digitale Anwendungen, die in allen Phasen der Cl-Versorgung zum Einsatz kommen können, von der Information und dem Screening potentieller Kandidaten über die präoperative Evaluation und Beratung, die Operation bis zur postoperativen Basis- und Folgetherapie und lebenslangen Nachsorge und klinischen Forschung.

„Artifical Intelligence“, „Cloud Connectivity“ und „Wireless Technology“ sind Begriffe, die zeigen, wohin die Reise bei der Cl-Versorgung geht bzw. heute schon Anwendung finden.

Viele Patienten informieren sich bereits heute im Vorfeld eines Arztbesuches und wünschen sich eine aktive Selbstbefähigung. Das wird durch Telemedizin und medizinische Apps ermöglicht. Vereinfachte Anpassungen (MAP-Erstellung) lassen sich z. B. durch eine (Semi-) Automatische MAP-Erstellung (z. B. NFS, FOX, bzw. andere Artificial Intelligence Anwendungen) realisieren. Durch Telemedizin, Remote Care Netzwerke und Apps rückt eine ortsnahe Versorgung der Cl-Träger in greifbare Nähe. Telemedizinische Konzepte erlauben hier völlig neue Formen der Patientenversorgung mit aktiver Beteiligung des Patienten, wie z. B. automatisierte technische Implantatkontrolle, Remote Care, Selbstprogrammierung und technologische Upgrades. In zentralen Datenbanken können u.a. die aktuelle MAP z. B. im Reparaturfall speichern und technische Daten und die Hörleistungen dokumentieren.

Einige der oben skizzierten Anwendungen sind heute schon Realität, andere erst in der Entwicklung.

Die bisherigen Entwicklungen zeigen, dass die Digitalisierung im Medizinsektor in rasanten Schritten vorangeht. Wenngleich noch viele Rahmenbedingungen und Details des Einsatzes digitaler Medi- en in der Medizin ausgestaltet werden müssen, ist diese Entwicklung absolut notwendig.

Bei der Betrachtung der Vorteile der Digitalisierung sollten auch Limitierungen diskutiert und Patienten und Akteure des Gesundheitswesens aktiv in diesen Prozess eingebunden werden. So sind z. B. Remote- und Self-Fitting nur für Patienten ohne spezielle audiologische und sonstige Besonderheiten einsetzbar.

Bezüglich der Sicherheit und des Datenschutzes in Deutschland - geregelt durch das Datenschutzrecht, das Telekommunikationsgesetz und das Telemediengesetz - wird es eine Herausforderung werden, dass der einzelne User stets die volle Kontrolle über seine Daten behält.

Die Diskussion um den digitalen Fortschritt kann dabei helfen, einen Rahmen für die digitale Ordnung zu finden, denn ohne die Expertise der Akteure im Gesundheitssystem wird auch mit „Artificial Intelligence“ eine Qualitätssicherung medizinischer Leistungen, zumindest in der nahen Zukunft, schwer möglich sein. Wir alle sind deshalb aufgefordert, diesen Prozess aktiv mitzugestalten. Für uns als HNO-Ärzte bedeutet das, dass wir neben einer hohen fachlichen Expertise auch hohen Anforderungen an die Qualifikation und Ausbildung in Bezug auf die digitalen Anwendungen gerecht werden müssen.

\section{Danksagung}

Die Autoren dieses Referates möchten sich bei allen bedanken, die durch vielfältigen Input, angeregte Diskussionen und Bereitstellung von Informationen und Abbildungen einen wichtigen Beitrag zu diesem Thema geleistet haben. Unser besonderer Dank geht an dieser Stelle an Cochlear, MED-EL und Advanced Bionics.

\section{Interessenkonflikt}

Die Autoren geben an, dass kein Interessenkonflikt besteht.

\section{Literature}

[1] WHO. Deafness and hearing loss. In; 2018

[2] Lin FR, Metter EJ, O'Brien RJ. et al. Hearing Loss and Incident Dementia. Archives of Neurology 2011; 68: 214-220

[3] Lin FR, Ferrucci L, An Y. et al. Association of hearing impairment with brain volume changes in older adults. Neurolmage 2014; 90

[4] Lin FR. Hearing loss and cognition among older adults in the United States. J Gerontol A Biol Sci Med Sci 2011; 66: 1131-1136

[5] Knopke S, Gräbel S, Förster-Ruhrmann U et al. Impact of cochlear implantation on quality of life and mental comorbidity in patients aged 80 years. Laryngoscope 2016; 126

[6] Olze H, Gräbel S, Förster $U$ et al. Elderly patients benefit from cochlear implantation regarding auditory rehabilitation, quality of life, tinnitus, and stress. The Laryngoscope 2012; 122: 196-203

[7] Olze H, Knopke S, Gräbel S et al. Rapid Positive Influence of Cochlear Implantation on the Quality of Life in Adults 70 Years and Older. Audiology and Neurotology 2016; 21

[8] Olze H, Gräbel S, Haupt $\mathrm{H}$ et al. Extra benefit of a second cochlear implant with respect to health-related quality of life and tinnitus. Otology \& neurotology: official publication of the American Otological. Society, American Neurotology Society [and] European Academy of Otology and Neurotology 2012; 33: 1169-1175 
[9] Knopke S, Szczepek A], Haussler SM et al. Cochlear Implantation of Bilaterally Deafened Patients with Tinnitus Induces Sustained Decrease of Tinnitus-Related Distress. Front Neurol 2017; 8: 158

[10] Hirschfelder A, Gräbel S, Olze H. The impact of cochlear implantation on quality of life: the role of audiologic performance and variables. Otolaryngology - head and neck surgery: official journal of American Academy of Otolaryngology-Head and Neck Surgery 2008; 138 : 357-362

[11] Olze H, Szczepek A], Haupt H et al. Cochlear implantation has a positive influence on quality of life, tinnitus, and psychological comorbidity. The Laryngoscope 2011; 121: 2220-2227

[12] Olze H, Szczepek A], Haupt H et al. The impact of cochlear implantation on tinnitus, stress and quality of life in postlingually deafened patients. Audiol Neurootol 2012; 17: 2-11

[13] DGHNO Pd. Weißbuch Cochlea-Implantat(Cl)-Versorgung.

[14] Blamey PJ, Maat B, Başkent D et al. A Retrospective Multicenter Study Comparing Speech Perception Outcomes for Bilateral Implantation and Bimodal Rehabilitation. Ear Hearing 2015; 36: 408-416

[15] Lenarz T. Cochlear Implant - State of the Art. Laryngorhinootologie 2017; 96: S123-S151

[16] Knopke S, Szczepek A], Häussler SM et al. Cochlear Implantation of Bilaterally Deafened Patients with Tinnitus Induces Sustained Decrease of Tinnitus-Related Distress. Frontiers in Neurology 2017; 8 : 158-158

[17] Disorders NloDaOC. Cochlear Implants. In; 2017

[18] Bundesamt S. Bevölkerung und Erwerbstätigkeit. 2015, DOI: 1-69

[19] Deutschen Gesellschaft für Hals-Nasen-Ohren-Heilkunde K-uH-CeV, Bonn. Cochlea-Implantat Versorgung und zentral-auditorische Implantate. In; 2012

[20] Knopke S, Olze H. Hörrehabilitation mithilfe von Cochleaimplantaten und kognitive Fähigkeiten. HNO 2017, doi:10.1007/s00106-017-0423-z

[21] Mathers C, Smith A, Concha M. Global burden of hearing loss in the year 2000 .

[22] Sohn W, Jorgenshaus W. Hals-Nasen-Ohren-Erkrankungen-Schwerhorigkeit in Deutschland-Reprasentative Horscreening-Untersuchung bei 2000 Probanden in 11 Allgemeinpraxen. ZFA-Zeitschrift fur Allgemeinmedizin-Ausgabe A 2001; 77: 143-147

[23] Teschner M, Polite C, Lenarz T et al. Cochlear implantation in different health-care systems: disparities between Germany and the United States. Otology \& Neurotology 2013; 34: 66-74

[24] Hoppe U, Hast A, Hocke T. Audiometry-based screening procedure for cochlear implant candidacy. Otology \& Neurotology 2015; 36: 1001-1005

[25] Sorkin DL. Cochlear implantation in the world's largest medical device market: utilization and awareness of cochlear implants in the United States. Cochlear implants international 2013; 14: S12-S14

[26] Bionics A. http://www.AdvancedBionics.de In; 2018

[27] Cochlear. www.cochlear.de In; 2018

[28] Medel. www.medel.com In; 2018

[29] Oticon. www.oticonmedical.com In; 2018

[30] Aiello CP, Ferrari DV. Teleaudiology: efficacy assessment of an online social network as a support tool for parents of children candidates for cochlear implant. In, CoDAS: SciELO Brasil 2015; 411-418

[31] Institute I. https://idainstitute.com/ In; 2018

[32] Paglialonga A, Nielsen AC, Ingo E. et al. eHealth and the hearing aid adult patient journey: a state-of-the-art review. Biomedical engineering online 2018; 17: 101

[33] Uecker FC, Szczepek A], Olze H. Paediatric bilateral cochlear implantation: simultaneous versus sequential surgery. Otol Neurotol. 2018 ; in press
[34] Hinderink JB, Krabbe PF, Van Den Broek P. Development and application of a health-related quality-of-life instrument for adult with cochlear implants: the Nijmegen cochlear implant questionnaire. Otolaryng Head Neck 2000; 123: 756-765

[35] Holube I, Kollmeier B. Modiflkation eines Fragebogens zur Erlassung des subjektiven Hör-verDlögens und dessen Beziehung zur Sprachverständlichkeit in Ruhe und unter Störgeräuschen. 1994

[36] Nasreddine ZS, Phillips NA, Bédirian V et al. The Montreal Cognitive Assessment, MoCA: a brief screening tool for mild cognitive impairment. Journal of the American Geriatrics Society 2005; 53 : 695-699

[37] Smits C, Theo Goverts S, Festen JM. The digits-in-noise test: assessing auditory speech recognition abilities in noise. The Journal of the Acoustical Society of America 2013; 133: 1693-1706

[38] Hoppe U, Hocke T, Hast A et al. Langzeitergebnisse eines Screeningverfahrens für erwachsene Cochlea-Implantat-Kandidaten. Laryngo-Rhino-Otologie 2017; 96: 234-238

[39] Bright T, Pallawela D. Validated smartphone-based apps for ear and hearing assessments: a review. JMIR rehabilitation and assistive technologies 2016; 3

[40] Nast DR, Speer WS, Le Prell CG. Sound level measurements using smartphone "apps": Useful or inaccurate? Noise and Health 2014; 16: 251

[41] Bruggemann P, Szczepek A], Klee K et al. In Patients Undergoing Cochlear Implantation, Psychological Burden Affects Tinnitus and the Overall Outcome of Auditory Rehabilitation. Front Hum Neurosci 2017; 11: 226

[42] Hinderink JB, Krabbe PF, Van Den Broek P. Development and application of a health-related quality-of-life instrument for adults with cochlear implants: the Nijmegen cochlear implant questionnaire. Otolaryngology - head and neck surgery: official journal of American Academy of Otolaryngology-Head and Neck Surgery 2000; 123: $756-765$

[43] Sweetow RW. Screening for cognitive disorders in older adults in the audiology clinic. Audiology Today 2015; 27: 38-43

[44] Morris MA, Saboury B, Burkett B et al. Reinventing radiology: big data and the future of medical imaging. Journal of thoracic imaging 2018; 33: 4-16

[45] Brink JA, Arenson RL, Grist TM et al. Bits and bytes: the future of radiology lies in informatics and information technology. European radiology 2017; 27: 3647-3651

[46] Radiology ESo. Summary of the proceedings of the international forum 2016:"Imaging referral guidelines and clinical decision support - how can radiologists implement imaging referral guidelines in clinical routine?". Insights into imaging 2017; 8: 1-9

[47] Kansagra AP, John-Paul JY, Chatterjee AR et al. Big data and the future of radiology informatics. Academic radiology 2016; 23: 30-42

[48] Gerber N, Bell B, Gavaghan K et al. Surgical planning tool for robotically assisted hearing aid implantation. International journal of computer assisted radiology and surgery 2014; 9: 11-20

[49] Lu P, Barazzetti L, Chandran V et al. Facial nerve image enhancement from $C B C T$ using supervised learning technique. In, Engineering in Medicine and Biology Society (EMBC), 2015 37th Annual International Conference of the IEEE: IEEE; 2015: 2964-2967

[50] Mellor JC, Stone MA, Keane J. Application of data mining to "big data" acquired in audiology: Principles and potential. Trends in hearing 2018; 22: 2331216518776817

[51] Cox S, Oakes M, Wermter S et al. AudioMine: Medical Data Mining in Heterogeneous Audiology Records. World Academy of Science, Engineering and Technology, International Journal of Computer, Electrical, Automation, Control and Information Engineering 2007; 1: $141-144$ 
[52] Czaplik M, Voigt V, Kenngott H et al. Why OR. NET? Requirements and perspectives from a medical user's, clinical operator's and device manufacturer's points of view. Biomedical Engineering/Biomedizinische Technik 2018; 63: 5-10

[53] Kasparick M, Schmitz M, Andersen B et al. OR. NET: a service-oriented architecture for safe and dynamic medical device interoperability. Biomedical Engineering/Biomedizinische Technik 2018; 63: 11-30

[54] Benzko J, Krause L, Janß A et al. Modular user interface design for integrated surgical workplaces. Biomedical Engineering/Biomedizinische Technik 2016; 61: 183-197

[55] Klenzner T, Ngan CC, Knapp FB et al. New strategies for high precision surgery of the temporal bone using a robotic approach for cochlear implantation. European archives of oto-rhino-laryngology 2009; 266: 955

[56] Caversaccio M, Gavaghan K, Wimmer W et al. Robotic cochlear implantation: surgical procedure and first clinical experience. Acta Oto-Laryngol 2017; 137: 447-454

[57] Cosetti MK, Troob SH, Latzman JM et al. An evidence-based algorithm for intraoperative monitoring during cochlear implantation. Otology \& Neurotology 2012; 33: 169-176

[58] Viccaro M, Covelli E, De Seta E et al. The importance of intra-operative imaging during cochlear implant surgery. Cochlear implants international 2009; 10: 198-202

[59] Vogl T, Tawfik A, Emam A et al. Pre-, intra-and post-operative imaging of cochlear implants. In, RöFo-Fortschritte auf dem Gebiet der Röntgenstrahlen und der bildgebenden Verfahren: (c Georg Thieme Verlag KG 2015; 980-989

[60] Carelsen B, Grolman W, Tange R et al. Cochlear implant electrode array insertion monitoring with intra-operative $3 \mathrm{D}$ rotational $\mathrm{X}$-ray. Clinical Otolaryngology 2007; 32: 46-50

[61] Aschendorff A. Imaging in cochlear implant patients. GMS current topics in otorhinolaryngology, head and neck surgery 2011; 10

[62] Bloom JD, Rizzi MD, Germiller JA. Real-time intraoperative computed tomography to assist cochlear implant placement in the malformed inner ear. Otology \& Neurotology 2009; 30: 23-26

[63] Yuan Y-Y, Song Y-S, Chai C-M et al. Intraoperative CT-guided cochlear implantation in congenital ear deformity. Acta Oto-Laryngol 2012; 132: 951-958

[64] Stelter K, Ledderose G, Hempel JM et al. Image guided navigation by intraoperative CT scan for cochlear implantation. Computer Aided Surgery 2012; 17: 153-160

[65] Shapiro WH, Huang T, Shaw T et al. Remote intraoperative monitoring during cochlear implant surgery is feasible and efficient. Otology \& Neurotology 2008; 29: 495-498

[66] Yanov Y, Kuzovkov V, Sugarova S et al. Successful application and timing of a remote network for intraoperative objective measurements during cochlear implantation surgery. International journal of audiology 2018 DOI: 1-7

[67] Botros A, van Dijk B, Killian M. AutoNRTTM: An automated system that measures ECAP thresholds with the Nucleus ${ }^{\circledR}$ Freedom ${ }^{\mathrm{TM}}$ cochlear implant via machine intelligence. Artificial Intelligence in Medicine 2007; 40: 15-28

[68] van Dijk B, Botros AM, Battmer R-D et al. Clinical results of AutoNRT, TM a completely automatic ECAP recording system for cochlear implants. Ear Hearing 2007; 28: 558-570

[69] Tavartkiladze G, Bakhshinyan V, Irwin C. Evaluation of new technology for intraoperative evoked compound action potential threshold measurements. International journal of audiology 2015; 54: 347-352

[70] Grolman W, Maat A, Verdam F et al. Spread of excitation measurements for the detection of electrode array foldovers: a prospective study comparing 3-dimensional rotational x-ray and intraoperative spread of excitation measurements. Otology \& Neurotology 2009; 30: $27-33$
[71] Lassaletta L, Polak M, Huesers J et al. Usefulness of Electrical Auditory Brainstem Responses to Assess the Functionality of the Cochlear Nerve Using an Intracochlear Test Electrode. Otology \& Neurotology 2017; 38: e413-e420

[72] „Vom“, DOI, 2018, 34

[73] Smoorenburg GF, Willeboer C, van Dijk JE. Speech perception in nucleus CI24M cochlear implant users with processor settings based on electrically evoked compound action potential thresholds. Audiol Neurootol 2002; 7: 335-347

[74] Battmer R-D, Borel S, Brendel M et al. Assessment of 'Fitting to Outcomes Expert' FOX ${ }^{\mathrm{TM}}$ with new cochlear implant users in a multi-centre study. Cochlear Implants International 2015; 16: 100-109

[75] Meeuws M, Pascoal D, Bermejo I et al. Computer-assisted CI fitting: Is the learning capacity of the intelligent agent FOX beneficial for speech understanding? Cochlear Implants Int 2017; 18: 198-206

[76] Vaerenberg B, Govaerts PJ, de Ceulaer G et al. Experiences of the use of FOX, an intelligent agent, for programming cochlear implant sound processors in new users. Int J Audiol 2011; 50: 50-58

[77] Botros A, Banna R, Maruthurkkara S. The next generation of Nucleus (R) fitting: a multiplatform approach towards universal cochlear implant management. Int J Audiol 2013; 52: 485-494

[78] Lenarz T. Cochlear implant - state of the art. GMS Curr Top Otorhinolaryngol Head Neck Surg 2017; 16: Doc04

[79] Eikelboom RH, Jayakody DMP, Swanepoel DW et al. Validation of remote mapping of cochlear implants. J Telemed Telecare 2014; 20 : $171-177$

[80] Kuzovkov V, Yanov Y, Levin S et al. Remote programming of MED-EL cochlear implants: users' and professionals' evaluation of the remote programming experience. Acta Oto-Laryngologica 2014; 134 : 709-716

[81] Wasowski A, Skarzynski PH, Lorens A et al. Remote fitting of cochlear implant system. Cochlear implants international 2010; 11 (Suppl 1): 489-492

[82] Frago LM, Canon S, de la Rosa EJ et al. Programmed cell death in the developing inner ear is balanced by nerve growth factor and insulin-like growth factor I. J Cell Sci 2003; 116: 475-486

[83] Bright T, Pallawela D. Validated Smartphone-Based Apps for Ear and Hearing Assessments: A Review. JMIR Rehabilitation and Assistive Technologies 2016; 3: e13-e13

[84] Bionics A. Die neue bimodale Naída Hörlösung.

[85] Bionics A. Die Naída Link CROS Lösung.

[86] Cullington H, Kitterick P, Weal M et al. Feasibility of personalised remote long-term follow-up of people with cochlear implants: a randomised controlled trial. BMJ open 2018; 8: e019640

[87] Infrastruktur BfVud. https://www.bmvi.de/DE/Themen/Digitales/ Breitbandausbau/Breitbandatlas-Karte/start.html In; 2018

[88] FAZ. http://www.faz.net/aktuell/wirtschaft/diginomics/so-weit-istdeutschland-mit-dem-breitbandausbau-15672719.html In; 2018

[89] Saunders GH, Chisolm TH. Connected audiological rehabilitation: $21^{\text {st }}$ century innovations. J Am Acad Audiol 2015; 26: 768-776

[90] Bush ML, Thompson R, Irungu C et al. The role of telemedicine in auditory rehabilitation: a systematic review. Otology \& neurotology: official publication of the American Otological Society, American Neurotology Society [and] European Academy of Otology and Neurotology 2016; 37: 1466

[91] Pichora-Fuller MK, Levitt H. Speech comprehension training and auditory and cognitive processing in older adults. American Journal of Audiology 2012; 21: 351-357

[92] Krüger-Brand HE. Mobile Medizintechnik: Telemedizin für die Nachsorge. Dtsch Arztebl International 2013; 110: 2014-2015 
[93] Cullington HE, Agyemang-Prempeh A. Person-centred cochlear implant care: Assessing the need for clinic intervention in adults with cochlear implants using a dual approach of an online speech recognition test and a questionnaire. Cochlear implants international 2017; 18: 76-88

[94] Zenner H, Delb W, Kröner-Herwig B et al. On the interdisciplinary S3 guidelines for the treatment of chronic idiopathic tinnitus. Hno 2015; 63: 419-427

[95] Folmer RL, Griest SE, Meikle MB et al. Tinnitus severity, loudness, and depression. Otolaryngology - Head and Neck Surgery 1999; 121: 48-51

[96] Kreuzer PM, Vielsmeier V, Langguth B. Chronic tinnitus: an interdisciplinary challenge. Deutsches Ärzteblatt International 2013; 110: 278

[97] Mazurek B, Szczepek A], Bruggemann P. Tinnitus - Clinical Symptoms and Therapy. Laryngorhinootologie 2017; 96: 47-59

[98] Henry JA, Dennis KC, Schechter MA. General review of tinnitus: prevalence, mechanisms, effects, and management. Journal of speech, language, and hearing research 2005; 48: 1204-1235
[99] Baguley DM, Atlas MD. Cochlear implants and tinnitus. Progress in brain research 2007; 166: 347-355

[100] Kompis M, Pelizzone M, Dillier $\mathrm{N}$ et al. Tinnitus before and 6 months after cochlear implantation. Audiology and Neurotology 2012; 17: $161-168$

[101] Miyamoto RT, Bichey BG. Cochlear implantation for tinnitus suppression. Otolaryngologic Clinics of North America 2003; 36: 345-352

[102] Pan T, Tyler RS, Ji H et al. Changes in the tinnitus handicap questionnaire after cochlear implantation. American journal of audiology 2009; 18: 144-151

[103] Quaranta N, Fernandez-Vega S, D'Elia C et al. The effect of unilateral multichannel cochlear implant on bilaterally perceived tinnitus. Acta Oto-Laryngol 2008; 128: 159-163

[104] Quaranta N, Wagstaff S, Baguley DM. Tinnitus and cochlear implantation. International journal of audiology 2004; 43: 245-251

[105] Olze H. Cochlear implants and tinnitus. HNO 2015; 63: 291-297 